OPEN ACCESS

Edited by:

M. Foster Olive,

Arizona State University, United States

Reviewed by:

Esa R. Korpi,

University of Helsinki, Finland

Somchai Amornyotin,

Mahidol University, Thailand

${ }^{*}$ Correspondence:

Gavin J. Kilpatrick

gavinkilpatrick@gjkpharma.co.uk

Specialty section: This article was submitted to

Neuropharmacology,

a section of the journal

Frontiers in Pharmacology

Received: 04 April 2021

Accepted: 06 July 2021

Published: 20 July 2021

Citation:

Kilpatrick GJ (2021) Remimazolam: Non-Clinical and Clinical Profile of a

New Sedative/Anesthetic Agent.

Front. Pharmacol. 12:690875

doi: 10.3389/fphar.2021.690875

\section{Remimazolam: Non-Clinical and Clinical Profile of a New Sedative/Anesthetic Agent}

\author{
Gavin J. Kilpatrick* \\ GJK Pharma Ltd., Eltisley, United Kingdom
}

A program to identify novel intravenous sedatives with a short and predictable duration of action was initiated in the late 1990's by Glaxo Wellcome. The program focussed on the identification of ester-based benzodiazepine derivatives that are rapidly broken down by esterases. Remimazolam was identified as one of the lead compounds. The project at Glaxo was shelved for strategic reasons at the late lead optimization stage. Via the GSK ventures initiative, the program was acquired by the small biotechnology company, TheraSci, and, through successive acquisitions, developed as the besylate salt at CeNeS and PAION. The development of remimazolam besylate has been slow by industry standards, primarily because of the resource limitations of these small companies. It has, however, recently been approved for anesthesia in Japan and South Korea, procedural sedation in the United States, China, and Europe, and for compassionate use in intensive care unit sedation in Belgium. A second development program of remimazolam was later initiated in China, using a slightly different salt form, remimazolam tosylate. This salt form of the compound has also recently been approved for procedural sedation in China. Remimazolam has the pharmacological profile of a classical benzodiazepine, such as midazolam, but is differentiated from other intravenous benzodiazepines by its rapid conversion to an inactive metabolite resulting in a short onset/offset profile. It is differentiated from other intravenous hypnotic agents, such as propofol, by its low liability for cardiovascular depression, respiratory depression, and injection pain. The benzodiazepine antagonist flumazenil can reverse the effects of remimazolam in case of adverse events and further shorten recovery times. The aim of this review is to provide an analysis of, and perspective on, published non-clinical and clinical information on 1) the pharmacology, metabolism, pharmacokinetics, and pharmacodynamic profile of remimazolam, 2) the profile of remimazolam compared with established agents, 3) gaps in the current understanding of remimazolam, 4) the compound's discovery and development process and 5) likely future developments in the clinical use of remimazolam.

Keywords: remimazolam, sedation, anesthesia, benzodiazepine, midazolam, propofol, total intravenous anesthesia, short-acting anesthetic 


\section{INTRODUCTION}

\section{Intravenous Benzodiazepines}

The first benzodiazepine with "tranquilizing" properties, chlordiazepoxide, was synthesized in the 1950's (Sternbach, 1979). Its discovery led to a large number of derivatives, including many compounds that have been developed and marketed as drugs. Benzodiazepines bind to an extracellular site at the interface of the $\alpha$ and $\gamma$ subunits of the gamma-aminobutyric acid type $\mathrm{A}\left(\mathrm{GABA}_{\mathrm{A}}\right)$ receptor to modulate the activity of the brain's major inhibitory neurotransmitter, GABA (Sigel and Ernst, 2018; Kim and Hibbs, 2021). Most benzodiazepines induce a positive allosteric modulation of this ligand-gated chloride ion channel, resulting (usually) in neuronal hyperpolarization and inhibition of activity. Compounds acting at this site have been widely used as anxiolytics and sedatives and to induce muscle relaxation, amnesia, sleep, and anticonvulsive effects (Haefely et al., 1993).

The low aqueous solubility of most benzodiazepines means that intravenous formulations are problematic. Nevertheless, intravenous formulations of several benzodiazepines, including diazepam and lorazepam (Figure 1), have been developed for procedural sedation and anesthesia (Cornett et al., 2018); however, they have a long duration of action and can cause injection pain and thrombosis at the site of injection (Hegarty and Dundee, 1977). A significant development in the field of intravenous benzodiazepines was midazolam's introduction in
1982 as a water-soluble benzodiazepine (Figure 1) with a short duration of action (Dundee et al., 1984; Kanto, 1985). Midazolam is used widely as an intravenous agent to provide sedation for diagnostic and interventional procedures and longer-term sedation, including intensive care. However, midazolam has shortcomings. Most importantly, recovery is relatively slow and can be extended due to metabolism by cytochrome P450 3A4 and the production of active metabolites (Bauer et al., 1995). The dependence on cytochrome P450 3A4 means that drug-drug interactions can be problematic since many drugs inhibit this enzyme (Yuan, Flockhart and Balian, 1999).

\section{Propofol}

Propofol (Figure 1) was discovered by ICI (now part of Astra Zeneca) (Glen and Hunter, 1984) and approved for the induction and maintenance of anesthesia in 1986. The precise mechanism of action of propofol is not fully understood, but it is thought to mediate much of its effects by positive modulation of the $\mathrm{GABA}_{\mathrm{A}}$ receptor. This is the same receptor where benzodiazepines act, but propofol is thought to act at a different site, the $\beta_{3}$ subunit (Jurd et al., 2003). It is not soluble in water and is formulated as a lipid-based emulsion. Propofol is the most widely used intravenous anesthetic drug globally and has revolutionized anesthetic practice and significantly improved patient care (Wood and Stark, 2018). Propofol has a very fast onset and offset of action. The short offset results from the redistribution<smiles>Cc1ncc2n1-c1ccc(Cl)cc1C(c1ccccc1F)=NC2</smiles> 
Remimazolam<smiles>COC(=O)CC[C@@H]1N=C(c2ccccn2)c2cc(Br)ccc2-n2c(C)cnc21</smiles>

CNS 7054<smiles>Cc1cnc2n1-c1ccc(Br)cc1C(c1ccccn1)=N[C@H]2CCC(=O)O</smiles>

\section{Benzene sulphonic acid Toluene sulphonic acid \\ (besylate) \\ (tosylate)}<smiles>O=S(=O)(O)c1ccccc1</smiles><smiles>Cc1ccc(S(=O)(=O)O)cc1</smiles>

FIGURE 2 | Remimazolam, salts of remimazolam, and the carboxylic acid metabolite, CNS 7054.

into lipid compartments rather than rapid metabolism (Simons et al., 1988).

The use of propofol is usually restricted to anesthetists because of its potential to induce profound sedative effects associated with hypotension, bradycardia, respiratory depression, and potentially apnea (Perel, 2011; Sahinovic et al., 2018). Other adverse events related to propofol include pain on injection, which can be severe (Tan and Onsiong, 1998). The local anesthetic lignocaine is often co- or preadministered to mitigate this (Kamal et al., 2021). The pain on injection induced by propofol is thought to be mediated by off-target interaction with the transient receptor potential receptors, TRPA1 and TRPV1, on sensory neurons (Cornett et al., 2008; Matta et al., 2008). Prolonged propofol infusion at high doses is also associated with a rare but potentially fatal syndrome termed propofol infusion syndrome (PRIS) (Bray, 1998; Hemphill et al., 2019).

\section{Ester-Based Short-Acting Drugs}

Short-acting drugs provide the advantage of predictable control of their effects. Drugs with ester groups that are rapidly broken down to inactive acid metabolites have been used widely in anesthesia (Birgenheier et al., 2020) to provide controllable sedation/anesthesia, neuromuscular blockade, analgesia, and to regulate heart rate. These include the short-acting opiate analgesic, remifentanil (Feldman et al., 1991), neuromuscular blockers such as mivacurium (Savarese et al., 1988), and the beta ${ }_{1}$ blockers esmolol (Erhardt et al., 1982) and landiolol (Iguchi et al., 1992). Further discovery programs of intravenous ester- based anesthetic short-acting drugs have been reported more recently, including the topic of this review, remimazolam, the propanidid derivative AZD-3043 (Egan et al., 2012), and cyclopropyl methoxycarbonyl metomidate (AB700) (Ge et al., 2012). The latter two compounds do not appear to have progressed beyond early clinical trials.

\section{The Short-Acting Benzodiazepine Discovery Project}

In the late 1990's, the team at Glaxo Wellcome, who had discovered remifentanil (Feldman et al., 1991), embarked on another short-acting drug program focused on benzodiazepines. The project aimed to identify novel sedatives with a short and predictable duration of action (Pacofsky et al., 2002; Stafford et al., 2002; Feldman, 2020). GW502056 (remimazolam, Figure 2) was selected as a potential lead compound for this project based on parameters including rapid onset of sedation, short duration of action, wide separation of activity from the acid metabolite, and aqueous solubility. Some limited data on other potential lead compounds have been published. For example, CNS 7259 (Kilpatrick and Tilbrook, 2006), also termed compound 11a (Stafford et al., 2002)) has a high affinity for the human $\mathrm{GABA}_{\mathrm{A}}$ receptor benzodiazepine site (Ki $6 \mathrm{nM}$ ), with the carboxylic acid metabolite being more than 400 times less active than the parent ester. Recovery from the loss of righting reflex in rodents was observed in under half the time of midazolam $(25-30 \mathrm{mg} / \mathrm{kg}$ i.v.). In the micropig, CNS 7259 (0.05-1 mg/kg i.v.) induced sedation 
with a fast onset and a shorter offset than midazolam. CNS 7529 was not taken forward because of low aqueous solubility (Stafford et al., 2002).

Glaxo Wellcome halted the short-acting benzodiazepine program at the late lead optimization stage for strategic reasons, and the project was shelved for 2-3 years. Via the GSK Ventures initiative, the program, including all the compounds, patents, and associated data, was subsequently acquired in an "asset for equity transaction" (assignment in exchange for shares) by the UK-based private biotechnology company, TheraSci Ltd. TheraSci was itself simultaneously acquired by the UK-based public company, CeNeS PLC. Further pre-clinical characterization at $\mathrm{CeNeS}$ confirmed remimazolam as the lead candidate, and development studies to support clinical trials commenced. The first public information on the pharmacological and pharmacokinetic characterization of remimazolam (then CNS 7056) was presented at the American Society of Anaesthesiologists meeting in 2006. A series of a platform presentation and three posters were presented (Kilpatrick et al., 2006a; Kilpatrick et al., 2006b; Mutter et al., 2006; Tilbrook and Kilpatrick, 2006), followed a year later by a full paper published in Anesthesiology (Kilpatrick et al., 2007). The development program of remimazolam, using the besylate salt, was continued by $\mathrm{CeNeS}$ until it was acquired by the German-based company PAION AG in 2008. An investigational new drug application was filed with the U.S. Food and Drug Administration in 2008, and the first phase I study in healthy volunteers was completed later that year. Further development was undertaken by PAION and its licensees. The international non-proprietary name (INN) of remimazolam was approved in 2010 (International Nonproprietary Names for Pharmaceutical Substances (INN), 2010).

Using a slightly different (tosylate) salt form of remimazolam (Figure 2), a second development program was later initiated by Jiangsu Hengrui Pharmaceutical Co. Ltd. in China. The selection of the different salt was presumably a maneuver to circumvent the existing patent protection of the compound's salts and polymorphic forms (Tilbrook and Bitt, 2006).

Remimazolam besylate has recently been approved for general anesthesia in Japan (Keam, 2020; Masui, 2020) and South Korea, procedural sedation in the United States (FDA, 2020b), China, and Europe (EMA, 2021). Compassionate use of remimazolam besylate for sedation in intensive care (ICU) has also been approved in Belgium (FAMHP, 2020). The tosylate salt has been approved for procedural sedation in China (Wang and Sun, 2020).

\section{REMIMAZOLAM}

Alternative names for remimazolam include, GW502056, CNS 7056, CNS 7056B, CNS 7056BS, ONO-2745, and HR 7056. The chemical name of the base is methyl 3-[(4S)-8-bromo-1-methyl-6-pyridin-2-yl$4 \mathrm{H}$-imidazo[1,2-a][1,4]benzodiazepin-4-yl] propanoate. Two salt forms have been developed: besylate and tosylate (Figure 2).
Remimazolam besylate is formulated as a lyophilized product for reconstitution (FDA, 2020a) but has sparing aqueous solubility, meaning that relatively large administration volumes need to be employed to accommodate the doses required in the anesthesia setting (Hirata et al., 2020).

\section{Pharmacology}

Remimazolam shows high affinity $(\mathrm{Ki} \sim 30 \mathrm{nM})$ for the $\mathrm{GABA}_{\mathrm{A}}$ receptor benzodiazepine site without significant activity at offtarget receptors, ion channels, or enzyme sites that have been tested (Kilpatrick et al., 2007). Remimazolam weakly inhibits the hERG tail current with estimated $\mathrm{EC}_{25}$ and $\mathrm{EC}_{50}$ values of 62 and $207 \mu \mathrm{M}$ (Kleiman et al., 2020). The compound showed positive modulation of the four major subtypes of the $\mathrm{GABA}_{\mathrm{A}}$ receptor that respond to benzodiazepines $\left(\alpha_{1}, a_{2}, \alpha_{3}, \alpha_{5}\right)$ and in that respect is very similar to "classical" benzodiazepines such as midazolam. In these electrophysiology studies, remimazolam showed slightly higher potency at the $\alpha_{1}$ subtype of the $\mathrm{GABA}_{\mathrm{A}}$ receptor $\left(\mathrm{EC}_{50}=\right.$ $0.36 \mu \mathrm{M})$. The $\alpha_{1}$ subtype is the site associated with sedative, anterograde amnesic, and anticonvulsant effects of benzodiazepines (Rudolph et al., 1999; McKernan et al., 2000; Rudolph and Möhler, 2004).

The carboxylic acid metabolite of remimazolam, CNS 7054, was 300-400 times weaker $(\mathrm{Ki} \sim 10,000 \mathrm{nM})$ at the benzodiazepine site of the $\mathrm{GABA}_{\mathrm{A}}$ receptor. CNS 7054 also showed no significant activity at off-target sites (Kilpatrick et al., 2007), and concentrations of up to $100 \mu \mathrm{M}$ did not inhibit the hERG tail current (Kleiman et al., 2020).

Rodent in vivo studies showed that remimazolam induced dose-related deep sedation with fast onset and offset (Kilpatrick et al., 2007). The offset was quicker than observed for midazolam. Pre-treatment with the benzodiazepine receptor antagonist, flumazenil (Hunkeler et al., 1981) inhibited the sedative effect of remimazolam. This finding supports the suggestion that its sedative effects were caused by activating the benzodiazepine site of the $\mathrm{GABA}_{\mathrm{A}}$ receptor. Inhibition of firing of neurons of the substantia nigra pars reticulata of the rat brain was also seen, with very rapid recovery after dosing ceased. This area of the brain receives prominent GABAergic innervation by striato-nigral afferents. The inhibition of firing observed provides further support for the $\mathrm{GABA}_{\mathrm{A}}$ receptor being the site of action (Kilpatrick et al., 2007).

Upton and colleagues conducted an extensive analysis of remimazolam's pharmacodynamics and pharmacokinetics in sheep (Upton et al., 2008, 2009; Upton et al., 2010). The pharmacokinetic studies are covered in Pharmacokinetics. The pharmacodynamic analysis revealed an apparent dose-related sedative effect of remimazolam (measured using EEG). There was a short duration of action without significant cardiovascular or respiratory depression (Upton et al., 2008; Upton et al., 2010). In a comparator study, remimazolam produced sedation with more rapid onset and offset rates than midazolam and propofol (Upton et al., 2009). The effects evoked by all three agents on the cardiovascular and respiratory systems were proportional to the sedation depth.

Only a relatively small number of studies of remimazolam pharmacology have been published in peer-reviewed journals in 


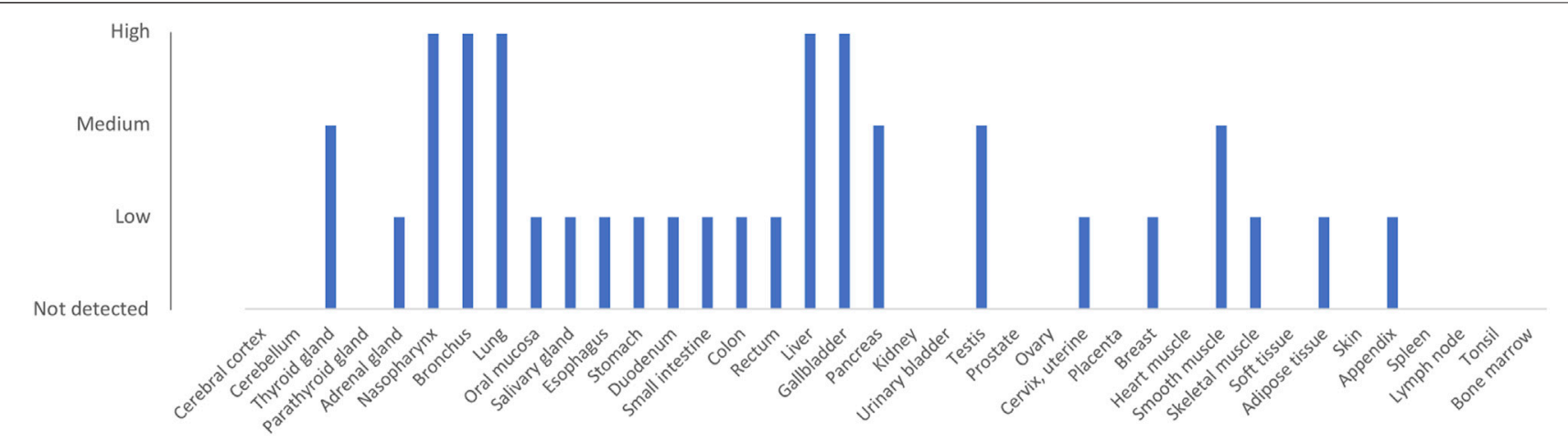

FIGURE 3 | Protein expression of CES1 (ENSG00000198848). Data are derived from the Human Protein Atlas database (Uhlén et al., 2015; CES1 protein expression, 2021b). Protein was detected using immunocytochemistry with antibodies HPA012023 and HPA046717.

the decade since the early work described above. Liu and colleagues describe the sedative profile of remimazolam and some closely related derivatives in rats and rabbits (Liu et al., 2016). Bevans and colleagues (Bevans et al., 2017) reported that remimazolam could induce sedation in mice via the inhalation route and that remimazolam potentiated the effects of remifentanil when both drugs were administered by that route to rats. Io et al. (Io et al., 2021) observed tolerance to remimazolam over a 28 days dosing period in the pig. However, the tolerance observed (assessed by the requirement to increase the dose to maintain the same sedative effect) was less than that seen with midazolam. Information on the sedative effects of acute bolus doses and short infusions of remimazolam in the pig is also provided, revealing a fast onset and offset profile. Kops et al. (Kops et al., 2021) describe dose-related sedative effects of remimazolam in the cynomolgus monkey with rapid onset. They also report strong synergism between the effects of remimazolam and remifentanil. Watanabe et al. (Watanabe et al., 2021) examined the actions of remimazolam on the ryanodine 1 receptor and the variant associated with malignant hyperthermia in response to volatile anesthetics. No effects associated with the ryanodine1 receptor were observed, and the authors conclude that remimazolam is unlikely to induce malignant hyperthermia.

Separate from the sedative effect of remimazolam, it has been reported (Xu et al., 2021) that derivatives of remimazolam induce apoptosis in glioma cells via down-regulation of the NF- $\kappa \mathrm{B}$ pathway. The mechanism involved is not clear. It is also not clear whether remimazolam itself has these effects; no data appear to be presented relating to remimazolam itself, although it is implied by the title of this publication. Recently, it has also been reported that remimazolam alleviates neuropathic pain via regulating the bradykinin $B_{1}$ receptor and autophagy (Xie et al., 2021). Studies to examine inhibition by flumazenil and the effects of other benzodiazepines, such as midazolam, would be useful in order to determine whether this effect is mediated via the benzodiazepine site on the $\mathrm{GABA}_{\mathrm{A}}$ receptor or some other effect of remimazolam or its metabolite.

While there are few published papers on the non-clinical characterization of remimazolam, many studies are known to have been conducted to support the regulatory filings. Some of the data from these are available publicly. For example, summaries of very extensive pharmacology, safety pharmacology, pharmacodynamic, drug interaction, and toxicology studies of remimazolam besylate are available on the Food and Drug Administration (FDA) website and the Japanese Pharmaceutical and Medical Devices Agency (PMDA) website, albeit in partially redacted form (Sokolowski, 2019; Mundipharma Co. Ltd., 2020).

\section{Metabolism}

Esterase metabolism breaks remimazolam down to its carboxylic acid metabolite, CNS 7054 (Figure 2), and methanol. Early studies showed that remimazolam (base; CNS 7056X) (Tilbrook and Kilpatrick, 2006) was very rapidly metabolized to the acid metabolite CNS 7054 by homogenates of liver from human, rat, mouse, and mini-pig. Rapid metabolism was also observed in tissues other than the liver, including mini-pig and rat kidney and rat lung. CNS 7056X was stable in plasma from human, pig, and dog for at least $60 \mathrm{~min}$. The breakdown of remimazolam largely via tissue esterases differentiates it from many other short-acting ester drugs, where blood esterases usually play a predominant role (e.g., remifentanil (Feldman et al., 1991) and esmolol (Erhardt et al., 1982)).

The major esterase responsible for remimazolam metabolism is often reported to be CES1 (Freyer et al., 2019; Sneyd and RigbyJones, 2020; Zhou et al., 2020a). However, no data appears to have been published to support this to date. Carboxylesterase 1 (CES1) is responsible for the metabolism of a number of drugs, including methylphenidate, clopidogrel, and the conversion of angiotensinconverting enzyme inhibitor prodrugs to the active drug (Laizure et al., 2013). It is highly expressed in the liver, gallbladder, and lung (Figure 3) (Uhlén et al., 2015; CES1 protein expression, 2021b). The genotype-tissue expression (GTEX) gene expression database (Carithers et al., 2015) (V8) shows that the liver is the organ with the highest expression, with a median number of CES1 transcripts per million (TPM) of 403 (from 226 individuals). The lung is the tissue showing the second-highest expression in the human body with 180 TPM. Other tissues with more than 50 TPM include the colon, major arteries, and adipose tissue (CES1 gene expression, 2021a). Transcript expression of 
TABLE 1 | Population pharmacokinetic (PK) and pharmacodynamic (PD) models of remimazolam. MOAA/S = modified observer's assessment of alertness and sedation. $\mathrm{BIS}=$ bispectral index. EEG = electroencephalogram. NA = not available/applicable. *JapicCTI-111495 not included in this study. Note that data from some of the studies referenced by Trial ID have not yet been published in full.

\begin{tabular}{|c|c|c|c|c|c|}
\hline Analysis & Trial ID & Salt form & PD measure & PK model & References \\
\hline PK/PD & CNS 7056-001 & Besylate & MOAA/S, BIS & $\begin{array}{l}\text { Physiologically based } \\
\text { recirculation }\end{array}$ & Wiltshire et al. (2012) \\
\hline PK/PD & NCT01970072 & Tosylate & MOAA/S, BIS & Three compartment & Zhou et al. (2018) \\
\hline PK/PD & EudraCT 2017-000455-12 & Besylate & MOAA/S & Three compartment & Schüttler et al. (2020) \\
\hline PD & EudraCT 2017-000455-12 & Besylate & EEG, MOAA/S & Three compartment & Eisenried et al. (2020) \\
\hline PD & $\begin{array}{l}\text { JapicCTI111495 } \\
\text { NCT01937767 } \\
\text { JapicCTI121973 } \\
\text { NCT03661489 }\end{array}$ & Besylate & Loss of consciousness, BIS, Narcotrend & $\mathrm{NA}$ & Lohmer et al. (2020) \\
\hline PK/PD & CNS 7056-001 & Besylate & $\mathrm{BIS}$ & Three compartment & Zhou et al., (2020a) \\
\hline PK & ONO-2745-01 & Besylate & NA & Three compartment & Zhou et al., (2020b) \\
\hline PK/PD, Markov* & $\begin{array}{l}\text { ONO-2745-02 } \\
\text { NCT01790607 } \\
\text { EudraCT 2017- } \\
\text { 000455-12 CNS } \\
\text { 7056-002 } \\
\text { NCT01145222 } \\
\text { NCT02290873 } \\
\text { JapicCTI111495 } \\
\text { NCT02296892 } \\
\text { NCT02532647 }\end{array}$ & Besylate & MOAAVS & Three compartment & Zhou et al. (2021) \\
\hline
\end{tabular}

CES1 in whole blood is relatively low (12 TPM). Overall, while expression of the protein and mRNA transcripts of CES1 is highest in the liver, the high expression in other tissues, including lung, suggests that extra-hepatic esterase metabolism of remimazolam (to CNS 7054) may be significant.

Significant variation in CES1 activity has been observed, and several genetic variants of CES1 associated with impaired enzyme activity have been identified (Wang et al., 2017; Gabriele et al., 2018). Individuals carrying these variants may be at risk of extended effects of remimazolam and other drugs metabolized by this enzyme. Extended effects of remimazolam have occasionally been observed (Zhou et al., 2020a; Yamamoto et al., 2021b), and further evaluation of the correlation of genetic variations in CES1 and the pharmacokinetics of remimazolam are warranted as well as interaction studies with other drugs known to be metabolized by this enzyme. Alcohol is a known inhibitor of CES1 (Parker et al., 2015) and is reported to increase the exposure $\left(\mathrm{C}_{\max }\right)$ to remimazolam by $1.2-2.1$ fold in a dose-dependent manner (Pesic et al., 2020b).

In an additional analysis to their phase I study in Chinese subjects (Sheng et al., 2020), an evaluation of the effect of genetic single nucleotide polymorphisms in the vitamin $\mathrm{D}$ receptor, cytochrome P450 3A4, and 3A5, as well as cytochrome P450 oxidoreductase on the pharmacokinetics of remimazolam and CNS 7054 was conducted (Hu et al., 2021). A small effect of the rs4516035 and rs1544410 variants in the vitamin D receptor on the pharmacokinetics of CNS 7054 and remimazolam, respectively, was found. The authors note that this was a small study, and further data are required.

Zhou et al. (Zhou et al., 2017) evaluated the metabolite profile of remimazolam (tosylate) in plasma and urine after intravenous administration to man. $2 \mathrm{~h}$ after administration, the relative percentage area in mass spectrometry analysis of plasma was $0.33 \%$ remimazolam, $99.67 \%$ CNS 7054, and no other metabolites were detected. In urine, after $4 \mathrm{~h}$, the percentages were $0.27 \%$ remimazolam and $98.63 \%$ CNS 7054 . The remaining $\sim 1 \%$ consisted of two oxidation products and two glucuronidation products of CNS 7054. Sheng et al. (Sheng et al., 2020) report that after an acute dose of remimazolam, almost no parent compound was recovered in the urine (0-24 h), but the percentage of CNS 7054 recovered in the urine was between 70 and 90 . These data support the hypothesis that the primary route of remimazolam metabolism is via esterases and that CYP enzymes do not play a significant role.

\section{Pharmacokinetics}

Early studies in a pig model revealed that remimazolam $(1.5 \mathrm{mg} / \mathrm{kg} / \mathrm{h}$ for $15 \mathrm{~min}$, i.v.) had a short half-life (18 min), a small volume of distribution (Vss $440 \mathrm{ml} / \mathrm{kg}$ ), and very rapid clearance $(35 \mathrm{ml} / \mathrm{min} / \mathrm{kg}$ ) (Mutter et al., 2006). Richard Upton's group presented detailed pharmacokinetic data from doseranging (i.v.) pharmacokinetic/pharmacodynamic studies in the sheep in 2010 (Upton et al., 2010). High clearance, small distribution volumes, and a rapid onset and offset of sedation with predictable effects over a wide dose range were observed. The kinetics of arterial sampling of CNS 7056 was linear with dose and described by a three-compartment model (volumes: 1.9, 3.9 , and $79 \mathrm{~L}$ ) with clearance of $4.2 \mathrm{~L} / \mathrm{min}$ and clearances between the compartment of 2.85 and $1.44 \mathrm{~L} / \mathrm{min}$. A mean residence time of $8.1 \mathrm{~min}$ was calculated for remimazolam. The $\mathrm{t}_{1 / 2, \mathrm{Ke} 0}$ (time for the concentration in the effect compartment to achieve $50 \%$ of the concentration in plasma) was $1.78 \mathrm{~min}$. 
TABLE 2 $\mid$ Published studies of remimazolam trials in human volunteers. $\mathrm{NA}=$ not available/applicable, PK = pharmacokinetics, $\mathrm{PD}=$ pharmacodynamics, iv = intravenous, $E C G$ = electrocardiogram. $N=$ number of subjects. *Note that the doses appear to represent the molecular weight of the salt and not to been corrected to base in these studies (Zhou et al., 2018).

\begin{tabular}{|c|c|c|c|c|c|c|c|c|c|}
\hline Trial ID & $\mathbf{N}$ & Endpoints & Salt & Dose & Route & $\begin{array}{l}\text { Infusion } \\
\text { duration }\end{array}$ & $\begin{array}{c}\text { Co- } \\
\text { treatment }\end{array}$ & Comparator & Ref \\
\hline CNS 7056-001 & 81 & Safety, PK, PD & Besylate & $0.01-0.3 \mathrm{mg} / \mathrm{kg}$ & iv & $1 \mathrm{~min}$ & NA & $\begin{array}{l}\text { Placebo, } \\
\text { midazolam }\end{array}$ & $\begin{array}{l}\text { Antonik et al. (2012); } \\
\text { Wiltshire et al. (2012) }\end{array}$ \\
\hline CNS 7056-002 (A) & 6 & Flumazenil reversal & Besylate & $0.25 \mathrm{mg} / \mathrm{kg}$ & iv & $1 \mathrm{~min}$ & Flumazenil & Placebo & $\begin{array}{l}\text { Worthington et al. } \\
\text { (2013) }\end{array}$ \\
\hline CNS 7056-002 (B) & 45 & $\begin{array}{l}\text { Colonoscopy: } \\
\text { Safety, efficacy }\end{array}$ & Besylate & $\begin{array}{l}0.04-0.1 \mathrm{mg} / \mathrm{kg}+ \\
\text { top-ups }\end{array}$ & iv & $1 \mathrm{~min}$ & Fentanyl & NA & $\begin{array}{l}\text { Worthington et al. } \\
\text { (2013) }\end{array}$ \\
\hline ONO-2745-01 & 42 & Safety, PK, PD & Besylate & $0.05-0.5 \mathrm{mg} / \mathrm{kg}$ & iv & $1 \mathrm{~min}$ & NA & NA & Doi, (2014) \\
\hline ONO-2745-02 & 10 & Safety, PK, PD & Besylate & $1 \mathrm{mg} / \mathrm{kg} / \mathrm{h}$ & iv & $\begin{array}{l}\text { Up to } \\
60 \mathrm{~min}\end{array}$ & NA & NA & Doi, (2014) \\
\hline $\begin{array}{l}\text { ChiCTR } \\
1800015185\end{array}$ & 62 & Safety, PK, PD & Besylate & $0.025-0.4 \mathrm{mg} / \mathrm{kg}$ & iv & $1 \mathrm{~min}$ & NA & $\begin{array}{l}\text { Placebo, } \\
\text { midazolam }\end{array}$ & Sheng et al., (2020) \\
\hline $\begin{array}{l}\text { ChiCTR } \\
1800015186\end{array}$ & 12 & Safety, PK, PD & Besylate & $\begin{array}{l}0.2 \mathrm{mg} / \mathrm{kg} \text { then } \\
1 \mathrm{mg} / \mathrm{kg} / \mathrm{h}\end{array}$ & iv & $1 \mathrm{~min}, 2 \mathrm{~h}$ & NA & Midazolam & Sheng et al., (2020) \\
\hline NCT01970072 & 79 & Safety, PK, PD & Tosylate & $0.01-0.45 \mathrm{mg} / \mathrm{kg}^{\star}$ & iv & $1 \mathrm{~min}$ & NA & $\begin{array}{l}\text { Placebo, } \\
\text { midazolam }\end{array}$ & Chen et al., (2020b) \\
\hline NCT03444480 & 8 & Flumazenil reversal & Tosylate & $\begin{array}{l}0.4 \mathrm{mg} / \mathrm{kg} \text { then } \\
1.5 \mathrm{mg} / \mathrm{kg} / \mathrm{h}^{\star}\end{array}$ & iv & $1 \mathrm{~min}, 2 \mathrm{~h}$ & Flumazenil & NA & Chen et al., (2020b) \\
\hline $\begin{array}{l}\text { EudraCT } 2017- \\
\text { 000455-12 }\end{array}$ & 20 & PK, PD & Besylate & $\begin{array}{l}5 \mathrm{mg} / \mathrm{min}, 3 \mathrm{mg} / \mathrm{min} \text {, } \\
1 \mathrm{mg} / \mathrm{min}\end{array}$ & iv & $\begin{array}{l}5,15 \\
15 \min \end{array}$ & NA & NA & Schüttler et al. (2020) \\
\hline NCT03329014 & 12 & Safety, PK, PD & Besylate & $10-40 \mathrm{mg}$ & Nasal & $\mathrm{NA}$ & NA & $\begin{array}{l}\text { Placebo, iv } \\
\text { remimazolam }\end{array}$ & Pesic et al., (2020a) \\
\hline NCT04113564 & 14 & Safety, PK, PD & Besylate & $0.14 \mathrm{mg} / \mathrm{kg}$ & Oral & NA & NA & iv remimazolam & Pesic et al. (2020b) \\
\hline $\begin{array}{l}\text { NCT04113343 } \\
\text { Part1 }\end{array}$ & 21 & Safety, PK, PD & Besylate & $60-480 \mathrm{mg}$ & Oral & NA & NA & NA & Pesic et al. (2020b) \\
\hline $\begin{array}{l}\text { NCT04113343 } \\
\text { Part2 }\end{array}$ & 11 & Safety, PK, PD & Besylate & $360 \mathrm{mg}$ & Oral & NA & Alcohol & NA & Pesic et al., (2020b) \\
\hline NCT04110535 & 40 & Abuse potential & Besylate & $5-10 \mathrm{mg}$ & iv & $1 \mathrm{~min}$ & NA & $\begin{array}{l}\text { Placebo, } \\
\text { midazolam }\end{array}$ & Schippers et al. (2020) \\
\hline CNS 7056-005 & 57 & ECG & Besylate & $10-20 \mathrm{mg}$ & iv & $1 \mathrm{~min}$ & NA & $\begin{array}{l}\text { Midazolam, } \\
\text { moxifloxacin, } \\
\text { placebo }\end{array}$ & Kleiman et al. (2020) \\
\hline $\begin{array}{l}\text { EudraCT } 2017- \\
\text { 000455-12 }\end{array}$ & 20 & ECG & Besylate & $\begin{array}{l}5 \mathrm{mg} / \mathrm{min}, 3 \mathrm{mg} / \mathrm{min} \text {, } \\
1 \mathrm{mg} / \mathrm{min}\end{array}$ & iv & $\begin{array}{l}5,15 \\
15 \min \end{array}$ & NA & Placebo & Kleiman et al. (2020) \\
\hline
\end{tabular}

The first human study of remimazolam (i.v.) included a detailed pharmacokinetic analysis from arterial and venous blood (Antonik et al., 2012). The pharmacokinetics of remimazolam were linear with dose, and its systemic clearance of $70.3 \mathrm{~L} / \mathrm{h}$ was approximately three times that seen with midazolam. The steady-state volume of distribution was $88.1 \mathrm{~L}$, terminal half-life $0.75 \mathrm{~h}$, and mean residence time $0.51 \mathrm{~h}$. Phase I single ascending dose trials of remimazolam besylate (Doi, 2014; Sheng et al., 2020) and remimazolam tosylate (Chen et al., 2020b) in Japanese and Chinese subjects report a similar profile to the initial phase I study with linear pharmacokinetics, high clearance, and short half-life. Pharmacokinetic analysis of remimazolam besylate administered by continuous infusions has been reported from volunteer studies (Sheng et al., 2020; Schüttler et al., 2020). The pharmacokinetic profiles were similar in both studies and consistent with those from single-dose studies.

The bioavailability of remimazolam after intranasal delivery of a powder formulation (10-40 mg) was $\sim 50 \%$ (Pesic et al., 2020a). Reduced bioavailability with increasing dose of solution formulation (47-26\%) was reported by the same authors, but this is explained as likely to be due to swallowing of excess fluid when larger volumes were employed. The calculated elimination half-lives of remimazolam after intranasal administration (10-40 mg, powder or solution) ranged from $0.7-1.2 \mathrm{~h}$, compared with $0.49 \mathrm{~h}$ after intravenous administration. When administered orally, the bioavailability of remimazolam is very low (1.1-2.2\%) (Pesic et al., 2020a). Elimination half-lives could be calculated, however. These ranged from $0.33-0.69 \mathrm{~h}$ (60-480 mg) compared with $0.44 \mathrm{~h}$ after intravenous administration (1.25-2.58 mg).

Analysis of the pharmacokinetics of the acid metabolite, CNS 7054, has usually been conducted simultaneously with that of remimazolam. A profile of a small volume of distribution together with a longer mean residence time and slower clearance compared to remimazolam is routinely reported in man and large animals (Mutter et al., 2006; Upton et al., 2010; Antonik et al., 2012; Pesic et al., 2020b; Pesic et al., 2020a; Schüttler et al., 2020; Sheng et al., 2020; Chen et al., 2020b). For example, Antonik et al. (Antonik et al., 2012) report an apparent clearance of 4.22 $\mathrm{L} / \mathrm{h}$, a volume of distribution of $17.5 \mathrm{~L}$, a half-life of $2.89 \mathrm{~h}$, and a mean residence time of 3.6-5.1 h. The pharmacokinetics of CNS 
TABLE 3 | Published clinical trials of remimazolam in procedural sedation. NA = not available/applicable, $\mathrm{Gl}=$ gastrointestinal. $\mathrm{N}=$ number of patients.

\begin{tabular}{|c|c|c|c|c|c|c|c|c|c|c|}
\hline Trial ID & Procedure & Phase & $\begin{array}{l}\text { ASA } \\
\text { class }\end{array}$ & $\mathbf{N}$ & $\begin{array}{l}\text { Primary } \\
\text { endpoint }\end{array}$ & $\begin{array}{l}\text { Salt } \\
\text { form }\end{array}$ & Dose & $\begin{array}{c}\text { Co- } \\
\text { treatment }\end{array}$ & Comparator & Ref \\
\hline NCT00869440 & $\begin{array}{l}\text { Upper Gl } \\
\text { endoscopy }\end{array}$ & $\|$ & $|-| \mid$ & 100 & $\begin{array}{l}\text { Success of } \\
\text { procedure }\end{array}$ & Besylate & $0.1-0.2 \mathrm{mg} / \mathrm{kg}$ & NA & Midazolam & $\begin{array}{l}\text { Borkett et al. } \\
(2015)\end{array}$ \\
\hline NCT01145222 & Colonoscopy & $\|$ & $|-|||$ & 162 & $\begin{array}{l}\text { Success of } \\
\text { procedure }\end{array}$ & Besylate & $\begin{array}{l}\text { 5-8 mg plus } 2-3 \mathrm{mg} \\
\text { top-ups }\end{array}$ & Fentanyl & Midazolam & $\begin{array}{l}\text { Pambianco } \\
\text { et al. (2016) }\end{array}$ \\
\hline NCT02290873 & Colonoscopy & III & I - III & 461 & $\begin{array}{l}\text { Success of } \\
\text { procedure }\end{array}$ & Besylate & $\begin{array}{l}5 \mathrm{mg} \text { plus } 2.5 \mathrm{mg} \\
\text { top-ups }\end{array}$ & Fentanyl & $\begin{array}{l}\text { Midazolam, } \\
\text { placebo }\end{array}$ & $\begin{array}{l}\text { Rex et al. } \\
(2018)\end{array}$ \\
\hline NCT02296892 & Bronchoscopy & III & I - III & 446 & $\begin{array}{l}\text { Success of } \\
\text { procedure }\end{array}$ & Besylate & $\begin{array}{l}5 \mathrm{mg} \text { plus } 2.5 \mathrm{mg} \\
\text { top-ups }\end{array}$ & Fentanyl & $\begin{array}{l}\text { Midazolam, } \\
\text { placebo }\end{array}$ & $\begin{array}{l}\text { Pastis et al. } \\
(2019)\end{array}$ \\
\hline NCT02532647 & Colonoscopy & III & III-IV & 79 & $\begin{array}{l}\text { Success of } \\
\text { procedure }\end{array}$ & Besylate & $\begin{array}{l}2.5-5.0 \mathrm{mg} \text { plus } \\
1.25-2.5 \mathrm{mg} \text { top-ups }\end{array}$ & Fentanyl & $\begin{array}{l}\text { Midazolam, } \\
\text { placebo }\end{array}$ & $\begin{array}{l}\text { Rex et al. } \\
(2021)\end{array}$ \\
\hline NCT03779061 & Colonoscopy & III & $|-| \mid$ & 384 & $\begin{array}{l}\text { Successful } \\
\text { sedation }\end{array}$ & Tosylate & $\begin{array}{l}5 \mathrm{mg} \text { plus } 2.5 \mathrm{mg} \\
\text { top-ups }\end{array}$ & Fentanyl & Propofol & $\begin{array}{l}\text { Chen et al., } \\
\text { (2020a) }\end{array}$ \\
\hline NCT03425474 & $\begin{array}{l}\text { Upper Gl } \\
\text { endoscopy }\end{array}$ & III & I - II & 384 & $\begin{array}{l}\text { Successful } \\
\text { sedation }\end{array}$ & Tosylate & $\begin{array}{l}5 \mathrm{mg} \text { plus } 2.5 \mathrm{mg} \\
\text { top-ups }\end{array}$ & Fentanyl & Propofol & $\begin{array}{l}\text { Chen et al. } \\
(2021)\end{array}$ \\
\hline $\begin{array}{l}\text { ChiCTR- } \\
2000038252\end{array}$ & Hysteroscopy & $\|$ & $|-| \mid$ & 82 & $\begin{array}{l}\text { Adverse } \\
\text { events }\end{array}$ & Besylate & $\begin{array}{l}0.2 \mathrm{mg} / \mathrm{kg} / \mathrm{min} \text { then } \\
1 \mathrm{mg} / \mathrm{kg} / \mathrm{h}\end{array}$ & Remifentanil & Propofol & Zhang et al. \\
\hline
\end{tabular}

\begin{tabular}{|c|c|c|c|c|c|c|c|c|c|c|}
\hline Trial ID & Procedure & Phase & $\begin{array}{l}\text { ASA } \\
\text { class }\end{array}$ & $\mathbf{N}$ & $\begin{array}{l}\text { Primary } \\
\text { endpoint }\end{array}$ & Salt & Dose & Co-treatment & Comparator & Ref \\
\hline JapicCTI111495 & $\begin{array}{l}\text { Various } \\
\text { surgeries }\end{array}$ & $\|$ & $|-| \mid$ & 85 & $\begin{array}{l}\text { Successful } \\
\text { anesthesia }\end{array}$ & Besylate & $\begin{array}{l}\text { Induction:Various } \\
\text { maintenance } \\
1 \mathrm{mg} / \mathrm{kg} / \mathrm{hr}\end{array}$ & $\begin{array}{l}\text { Remifentanil, } \\
\text { rocuronium }\end{array}$ & NA & $\begin{array}{l}\text { Doi (2014); Doi } \\
\text { et al. (2015) }\end{array}$ \\
\hline NCT01937767 & $\begin{array}{l}\text { Cardiac } \\
\text { surgery }\end{array}$ & $\|$ & NA & 90 & $\begin{array}{l}\text { Successful } \\
\text { anesthesia }\end{array}$ & Besylate & $\begin{array}{l}\text { Induction } \\
6-12 \mathrm{mg} / \mathrm{kg} / \mathrm{hr} \\
\text { maintenance } \\
\sim 1 \mathrm{mg} / \mathrm{kg} / \mathrm{hr}\end{array}$ & $\begin{array}{l}\text { Fentanyl/ } \\
\text { remifentanil, } \\
\text { rocuronium }\end{array}$ & $\begin{array}{l}\text { Propofol, } \\
\text { sevoflurane }\end{array}$ & $\begin{array}{l}\text { Probst et al. } \\
\text { (2014, 2015); } \\
\text { Bevilacqua et al. } \\
(2015)\end{array}$ \\
\hline JapicCTI121973 & $\begin{array}{l}\text { Various } \\
\text { surgeries }\end{array}$ & $\|/\| I \|$ & I- II & 375 & $\begin{array}{l}\text { Successful } \\
\text { anesthesia }\end{array}$ & Besylate & $\begin{array}{l}\text { Induction } \\
6-12 \mathrm{mg} / \mathrm{kg} / \mathrm{hr} \\
\text { maintenance } \\
\sim 1 \mathrm{mg} / \mathrm{kg} / \mathrm{hr} \text { (titration) }\end{array}$ & $\begin{array}{l}\text { Remifentanil, } \\
\text { rocuronium }\end{array}$ & Propofol & Doi et al., (2020a) \\
\hline NCT03661489 & $\begin{array}{l}\text { Various } \\
\text { surgeries }\end{array}$ & III & III & 67 & $\begin{array}{l}\text { Successful } \\
\text { anesthesia }\end{array}$ & Besylate & $\begin{array}{l}\text { Induction } \\
6-12 \mathrm{mg} / \mathrm{kg} / \mathrm{hr} \\
\text { maintenance } \\
2 \mathrm{mg} / \mathrm{kg} / \mathrm{hr}\end{array}$ & $\begin{array}{l}\text { Remifentanil, } \\
\text { muscle relaxant }\end{array}$ & NA & Doi et al., (2020b) \\
\hline
\end{tabular}

7054 has been reported to fit best to a two-compartment model in sheep (Upton et al., 2010) and man (with a transit compartment to account for metabolite formation) (Schüttler et al., 2020).

\section{Pharmacokinetic/pharmacodynamic Modeling}

A summary of PK/PD modeling analyses conducted with remimazolam, based on human data, is presented in Table 1. A physiologically based recirculation model was fitted to the pharmacokinetics of remimazolam from the initial clinical trial. In analyses since then, three-compartment models have been employed. Pharmacodynamic biomarker assessments have focused on EEG measures such as the bispectral index (BIS) score (Bower et al., 2000) and scores from the Modified Observer's Assessment of Alertness/Sedation (MOAA/S) scale (Chernik et al., 1990). Some of the reasons to switch to a 3- compartment pharmacokinetic model are logical, e.g., reduced complexity and to facilitate dosing technologies such as targetcontrolled infusion (Schüttler et al., 2020; Sneyd and Rigby-Jones, 2020). Other reasons for dismissing the Wiltshire model are less logical, e.g., to claim it is "discredited" (Zhou et al., 2020a) because it assumes lung metabolism. This is on the basis of the ratio of liver/lung CES1 mRNA selected from a small study (Gabriele et al., 2018). Zhou et al., do not present any empirical data and contradictory data from large datasets on protein and transcript expression exist showing a high level of expression of the CES1 gene/protein in the lung and other tissues (see Metabolism).

PK/PD modeling supports the observation of rapid onset and offset of effect. The $t_{1 / 2, \text { Keo }}$ parameter reflects the time required for the concentration in the effect compartment to reach $50 \%$ of the concentration in the plasma or the delay between cause and onset of effect. Calculated $t_{1 / 2, \mathrm{Ke} 0}$ values for remimazolam are usually reported in the range of $1-5 \mathrm{~min}$ (Wiltshire et al., 2012; Zhou 
TABLE 5 | Case reports on the use of remimazolam besylate in clinical practice. NA = Not available/applicable, ECRP = Endoscopic retrograde cholangiopancreatography, $\mathrm{MEP}=$ Motor evoked potentials, EEG = electroencephalograph.

\begin{tabular}{|c|c|c|c|c|c|}
\hline Procedure & Dose & Salt & Co-treatment & Comment & Ref \\
\hline Spine surgery & $\begin{array}{l}\text { Induction: } 6 \text { or } 12 \mathrm{mg} / \mathrm{kg} / \mathrm{h} \text {; } \\
\text { maintenance } \\
0.5-1.5 \mathrm{mg} / \mathrm{kg} / \mathrm{h}\end{array}$ & Besylate & $\begin{array}{l}\text { Remifentanil, } \\
\text { rocuronium }\end{array}$ & MEP monitoring by EEG. Successful & Kondo et al. (2020) \\
\hline Craniotomy & $\begin{array}{l}\text { Induction } 12 \mathrm{mg} / \mathrm{kg} / \mathrm{h} \text {; } \\
\text { maintenance } 1 \mathrm{mg} / \mathrm{kg} / \mathrm{h}\end{array}$ & Besylate & $\begin{array}{l}\text { Remifentanil, } \\
\text { fentanyl }\end{array}$ & $\begin{array}{l}\text { Awake when infusion stopped. } \\
\text { Successful }\end{array}$ & Sato et al. (2020) \\
\hline ECRP & $\begin{array}{l}\text { Induction } 12 \text { mg/kg/h; } \\
\text { maintenance } 1 \mathrm{mg} / \mathrm{kg} / \mathrm{h}\end{array}$ & Besylate & $\begin{array}{l}\text { Remifentanil, } \\
\text { rocuronium }\end{array}$ & $\begin{array}{l}\text { High risk: Myotonic dystrophy. } \\
\text { Successful }\end{array}$ & Fukuda et al. (2021) \\
\hline Cardiac surgery & $\begin{array}{l}\text { Induction } 6 \mathrm{mg} / \mathrm{kg} / \mathrm{h} \text {; } \\
\text { maintenance } \\
0.6-1 \mathrm{mg} / \mathrm{kg} / \mathrm{h}\end{array}$ & Besylate & $\begin{array}{l}\text { Remifentanil, } \\
\text { fentanyl, } \\
\text { rocuronium }\end{array}$ & $\begin{array}{l}\text { Cardiopulmonary bypass. } \\
\text { Successful }\end{array}$ & Saito et al. (2021) \\
\hline Laminoplasty for spinal stenosis & $\begin{array}{l}\text { Induction } 12 \text { mg/kg/h; } \\
\text { maintenance } 1 \mathrm{mg} / \mathrm{kg} / \mathrm{h}\end{array}$ & Besylate & Rocuronium & $\begin{array}{l}\text { Re-sleeping observed after } \\
\text { flumazenil reversal }\end{array}$ & $\begin{array}{l}\text { Yamamoto et al. } \\
\text { (2021b) }\end{array}$ \\
\hline Craniotomy & $\begin{array}{l}\text { Induction } 6 \mathrm{mg} / \mathrm{kg} / \mathrm{h} \text {; } \\
\text { maintenance } \\
0.75-1 \mathrm{mg} / \mathrm{kg} / \mathrm{h}\end{array}$ & Besylate & $\begin{array}{l}\text { Remifentanil } \\
\text { flumazenil }\end{array}$ & $\begin{array}{l}\text { Awakening during surgery with } \\
\text { flumazenil. Successful }\end{array}$ & $\begin{array}{l}\text { Yoshida et al., } \\
\text { (2021b) }\end{array}$ \\
\hline Craniotomy & NA & Besylate & NA & $\begin{array}{l}\text { Concerns around pharyngeal reflex, } \\
\text { blood pressure, and agitation on } \\
\text { flumazenil reversal }\end{array}$ & $\begin{array}{l}\text { Tachibana, } \\
\text { Hayamizu and } \\
\text { Yamakage, (2021) }\end{array}$ \\
\hline $\begin{array}{l}\text { Surgery: 1) stent removal and 2) tumor } \\
\text { resection }\end{array}$ & $\begin{array}{l}\text { Initially } 10 \text { and } 13 \text { mg bolus } \\
\text { respectively plus additional } \\
\text { doses }\end{array}$ & Besylate & $\begin{array}{l}\text { Remifentanil/ } \\
\text { fentanyl, } \\
\text { rocuronium }\end{array}$ & $\begin{array}{l}\text { Reduced effect in long-term } \\
\text { benzodiazepine users }\end{array}$ & $\begin{array}{l}\text { Yoshikawa et al. } \\
(2021)\end{array}$ \\
\hline $\begin{array}{l}\text { Cataract surgery, rigid laryngoscopy, } \\
\text { bronchoscopy, intramedullary nailing, and } \\
\text { hemiarthroplasty/hip fracture }\end{array}$ & $5-20 \mathrm{mg}$ & Besylate & NA & $\begin{array}{l}\text { Reduced impact on } \\
\text { neuropsychiatric function compared } \\
\text { to existing sedative agents }\end{array}$ & $\begin{array}{l}\text { Henson and } \\
\text { Thompson, (2021) }\end{array}$ \\
\hline Thyroid surgery & $\begin{array}{l}\text { Induction } 12 \text { mg/kg/h; } \\
\text { maintenance } 1 \mathrm{mg} / \mathrm{kg} / \mathrm{h}\end{array}$ & Besylate & $\begin{array}{l}\text { Remifentanil, } \\
\text { rocuronium }\end{array}$ & $\begin{array}{l}\text { Comparable anesthesia to existing } \\
\text { drugs for neuromonitoring during } \\
\text { thyroid surgery }\end{array}$ & $\begin{array}{l}\text { Hayamizu et al. } \\
(2021)\end{array}$ \\
\hline Hand surgery & Induction 6 mg/kg/h & Besylate & $\begin{array}{l}\text { Remifentanil, } \\
\text { fentanyl, } \\
\text { rocuronium }\end{array}$ & Anaphylaxis to remimazolam & Tsurumi et al. (2021) \\
\hline $\begin{array}{l}\text { MitraClip implantation in a patient with } \\
\text { advanced heart failure }\end{array}$ & $\begin{array}{l}\text { Induction } 6 \mathrm{mg} / \mathrm{kg} / \mathrm{h} \text {; } \\
\text { maintenance } 0.15 \mathrm{mg} / \mathrm{kg} / \mathrm{h}\end{array}$ & Besylate & $\begin{array}{l}\text { Remifentanil, } \\
\text { rocuronium }\end{array}$ & $\begin{array}{l}\text { Appropriate anesthetic management } \\
\text { in a patient with severe } \\
\text { cardiovascular disease }\end{array}$ & Satoh et al. (2021) \\
\hline $\begin{array}{l}\text { Cochlear implant in a patient with } \\
\text { mitochondrial encephalomyopathy }\end{array}$ & $\begin{array}{l}\text { Induction } 0.2 \mathrm{mg} / \mathrm{kg} \\
\text { (1 min); maintenance } \\
1 \mathrm{mg} / \mathrm{kg} / \mathrm{h}\end{array}$ & Besylate & Remifentanil & $\begin{array}{l}\text { Successful use for general } \\
\text { anesthesia in a patient with } \\
\text { mitochondrial myopathy }\end{array}$ & $\begin{array}{l}\text { Suzuki, Doi and } \\
\text { Nakajima, (2021) }\end{array}$ \\
\hline
\end{tabular}

et al., 2020a; Eisenried et al., 2020; Schüttler et al., 2020; Zhou et al., 2021). In a study of remimazolam tosylate, longer $t_{1 / 2, \mathrm{Ke} 0}$ values $\left(=\ln (2) / \mathrm{k}_{\mathrm{e} 0}\right)$ of 8.1 and $13.9 \mathrm{~min}$ were calculated for BIS monitoring and MOAA/S, respectively (Zhou et al., 2018). The reason for this difference from the other analyses is not clear.

A desirable feature in the anesthesia setting is that the elimination half time of a drug does not change significantly in relation to the duration of dosing. The context-sensitive halftime is a measure of this behavior, where the context is the duration of infusion. For remimazolam, the context-sensitive half-time appears to be largely insensitive to infusion duration, reaching a maximum after around $2 \mathrm{~h}$ at 6-11 min (Wiltshire et al., 2012; Eisenried et al., 2020; Schüttler et al., 2020). The predicted insensitivity of context-sensitive half-time with infusion duration calculated for remimazolam compares favorably to other hypnotics and opiates (Johnson, 2012).

Schüttler et al. (Schüttler et al., 2020) present calculated effect-site $\mathrm{EC}_{50}$ s for MOAA/S score from 4 (mild sedation: lethargic response to name spoken in normal tone) to 0 (unconscious: no response to painful trapezius squeeze) in the range of $337-1,579 \mathrm{ng} / \mathrm{ml}$. Remimazolam is $92 \%$ bound in plasma (Freyer et al., 2019). Data from brain homogenate binding does not appear to have been published, so corrections for bound/free compound cannot be made. The effect-site concentrations, uncorrected for bound drug, however, equate to concentrations of remimazolam of $\sim 0.77-3.6 \mu \mathrm{M}$, which compare with the observed $\mathrm{EC}_{50}$ of $0.36 \mu \mathrm{M}$ at the recombinant human $\alpha_{1} \mathrm{GABA}_{\mathrm{A}}$ receptor subtype generated from in vitro electrophysiology studies and the $\mathrm{Ki}$ of $0.03 \mu \mathrm{M}$ from radioligand binding assays (Kilpatrick et al., 2007).

Modeling to establish dosing schedules, with concomitant analgesic, in phase III procedural sedation and anesthesia trials and the marketed products has been conducted (Zhou et al., 2020a; Zhou et al., 2020b; Lohmer et al., 2020; Zhou et al., 2021). Dosing without correction for body weight is supported in procedural sedation (Wiltshire et al., 2012; Zhou et al., 2020b; Schüttler et al., 2020; Zhou et al., 2021). While some differences in the behavior of remimazolam are observed related to age, race, gender, obesity status, weight, and ASA class, covariate analysis indicates that major dose adjustments are not needed for the majority of patients (Zhou et al., 2018, 
2021; Zhou et al., 2020a; Zhou et al., 2020b; Lohmer et al., 2020).

\section{Clinical Data}

Multiple clinical trials of remimazolam have been conducted and published. They can be broadly divided into examining the effects of remimazolam in 1) human volunteers, 2) patients undergoing procedures under sedation, and 3) patients undergoing general anesthesia. These are summarized in Tables 2-4. Since remimazolam has reached the market, case reports of its clinical use are also starting to be published. These are summarized in Table 5. Several further remimazolam trials are registered on clinical trial databases; of note are studies examining its use for sedation in intensive care. However, the results of these are yet to be published.

\section{Volunteers}

The data from the first clinical trial of remimazolam in volunteers was reported in two papers published in 2012, one describing the safety, pharmacokinetics, and pharmacodynamics (Antonik et al., 2012) and the other, a population pharmacokinetic and pharmacodynamic modeling analysis (Wiltshire et al., 2012). The study evaluated nine single, ascending doses of remimazolam besylate $(0.01-0.3 \mathrm{mg} / \mathrm{kg})$ and included placebo and midazolam control groups. Doses were selected on the basis of the pre-clinical large animal data and modeling. As observed using BIS or MOAA/S score biomarkers, dose-related sedation with rapid onset and offset was seen in the remimazolam groups. The pharmacodynamic profile from large animals translated very well to humans, with the lowest dose that induced sedative effects being $0.075 \mathrm{mg} / \mathrm{kg}$ as was predicted by modeling (Antonik et al., 2012). The drug was well tolerated, with no supplemental oxygen or ventilation requirement. Times to recovery were shorter in the remimazolam treated groups than the midazolam treated group. Very similar single ascending dose studies of remimazolam besylate have been conducted in Japanese (Doi, 2014) and Chinese populations, one with remimazolam besylate (Sheng et al., 2020) and one with remimazolam tosylate (Chen et al., 2020b). These three studies had very similar outcomes of a good tolerability profile and dose-related sedation with rapid onset and offset. The study in Japanese subjects (Doi, 2014) included an elderly group ( $>65$ years), and no change in the profile associated with age was reported.

Infusion regimens of remimazolam have been evaluated in four volunteer studies, three using the besylate salt (Doi, 2014; Sheng et al., 2020; Schüttler et al., 2020) and one the tosylate (Chen et al., 2020b). In one study, a single dose of $1 \mathrm{mg} / \mathrm{kg} / \mathrm{h}$ for up to $60 \mathrm{~min}$ was used (Doi, 2014). The other three employed an initial higher dose $(0.2 \mathrm{mg} / \mathrm{kg} / \mathrm{min}$ [ $1 \mathrm{~min}$ ] (Sheng et al., 2020), $0.4 \mathrm{mg} / \mathrm{kg}$ [bolus] (Chen et al., 2020b), $5 \mathrm{mg} / \mathrm{min}$ [5 min] (Schüttler et al., 2020)) to induce sedation followed by a continuous infusion at a lower dose (1-2 mg/kg/h [2 h] (Sheng et al., 2020), $1.5 \mathrm{mg} / \mathrm{kg} / \mathrm{h}$ [2 h] (Chen et al., 2020b) and $3 \mathrm{mg} / \mathrm{min}$ [15 $\mathrm{min}$ ] followed by $1 \mathrm{mg} /$ min [15 min] (Schüttler et al., 2020). Sedation was monitored by BIS or EEG monitoring and MOAA/S scoring biomarkers.
These studies show a consistent theme of rapid onset of sedation, maintenance over the continuous infusion, and rapid recovery after the infusion was stopped.

Reversal of the sedation induced by remimazolam by flumazenil in a human study was first reported by Worthington et al., 2013 (Worthington et al., 2013), an observation that has since been confirmed in several studies (see Flumazenil reversal).

Further volunteer studies have been conducted to evaluate remimazolam's cardiac electrophysiology effects, its bioavailability by other administration routes, and its abuse potential (Kleiman et al., 2020; Pesic et al., 2020a; Pesic et al., 2020b). The latter two points are addressed in Adverse reactions and liabilities and Alternative indications and routes of administration. The authors of the cardiac electrophysiology studies conclude that remimazolam does not prolong cardiac repolarization although they observe that an increase in $\mathrm{QT}_{c}$ interval may occur after bolus administration resulting from an increase in heart rate (Kleiman et al., 2020).

The pharmacodynamic effects of remimazolam can be readily measured in volunteers, but data correlating these effects with target engagement at the $\mathrm{GABA}_{\mathrm{A}}$ benzodiazepine receptor are not available. Positron emission tomography technologies to measure in vivo binding to the benzodiazepine site of the $\mathrm{GABA}_{\mathrm{A}}$ receptor are well established, e.g., using $\left[{ }^{11} \mathrm{C}\right]$ or $\left[{ }^{18} \mathrm{~F}\right]$ derivatives of flumazenil (Persson et al., 1985; Gründer et al., 2001). It would be of interest to undertake studies to evaluate the benzodiazepine receptor engagement by remimazolam using this technique. The availability of such data may better inform modeling of the sedative actions of remimazolam.

\section{Procedural Sedation}

The sedation of patients to reduce pain, reduce anxiety and provide amnesia facilitates uncomfortable diagnostic procedures such as endoscopy (Dossa et al., 2021) and interventional procedures such as those employed in cardiology (Makkad, 2013). Several clinical trials of remimazolam as a sedative agent for procedural sedation have been conducted. The bulk have been in colonoscopy, upper gastrointestinal endoscopy, and bronchoscopy, and there is a single report of a trial in hysteroscopy (Table 3 ).

The first report of use in colonoscopy was a phase Ib doserange finding remimazolam besylate study in volunteers (Worthington et al., 2013). Multiple doses were employed in a design utilizing initial $(0.04-0.1 \mathrm{mg} / \mathrm{kg})$ and top-up doses to maintain sedation for $30 \mathrm{~min}$. Subjects also received a single dose of fentanyl. Successful sedation (judged by achieving a MOAA/S score of $\leq 4$ on three repeat measurements, completion of the procedure, no requirement for rescue sedative medication, and no requirement for assisted ventilation) was achieved in $>70 \%$ of subjects. Fast onset and offset of sedation were observed.

Three phase II studies of remimazolam besylate in procedural sedation have been published. The first was in 100 patients undergoing upper gastrointestinal endoscopy (Borkett et al., 2015), the second in 162 patients undergoing colonoscopy (Pambianco et al., 2016), and the third in 82 
patients undergoing hysteroscopy (Zhang et al., 2021). A midazolam-treated group was included as a comparator in the first two studies. In the third study, propofol was the comparator drug. In the upper G.I. endoscopy study, no analgesic co-treatment was reported to be employed. Relatively modest success rates were achieved (32\%, 56\%, and $64 \%$ for $0.10,0.15$ and $0.20 \mathrm{mg} / \mathrm{kg}$ remimazolam, respectively) compared with $44 \%$ of patients for midazolam $(0.075 \mathrm{mg} / \mathrm{kg})$. In the colonoscopy study, the analgesic fentanyl was co-administered, and higher success rates were observed ( $>92 \%$ for the remimazolam groups and $75 \%$ for the midazolam group). The hysteroscopy study's success rates were $100 \%$ for both the remimazolam and propofol groups, with a faster recovery seen in the remimazolam group. The remimazolam group's adverse event profile was superior to the propofol group in terms of hemodynamic fluctuation, excessive sedation depth, and low $\mathrm{SpO}$. There was a much lower incidence of injection pain in the remimazolam group.

Five phase III studies have been reported, three for remimazolam besylate and two for remimazolam tosylate. The doses routinely employed in the phase III studies were uncorrected for body weight and the same for both salts, i.e., an initial dose of $5 \mathrm{mg}$ with additional top-ups of $2.5 \mathrm{mg}$ as required.

The besylate studies are in colonoscopy (Rex et al., 2018, Rex et al., 2021) and bronchoscopy (Pastis et al., 2019) and include a colonoscopy study in a high-risk group (ASA class III-IV) (Rex et al., 2021). All three besylate studies were placebo-controlled with a midazolam comparator group (open label) and included co-treatment with the analgesic fentanyl. Success was defined as completion of colonoscopy, no need for an alternative sedative, or more than five top-ups in a 15 min period. Each trial met the primary endpoint, and the conclusion was reached for all studies that remimazolam was safe and effective for sedation during the procedures with rapid onset of effect and recovery. In these studies, the success rates in the remimazolam groups (80.6-91.3\%) were considerably higher than the midazolam (13.3-32.9\%) or placebo groups (0-4.8\%). In all studies, the procedure was started sooner in the remimazolam treatment arm (5.1-8.0 min) than in the midazolam (13.3-32.9 min) or placebo control arms $(12-21.9 \mathrm{~min})$. The time to return to full alertness after the procedure was shorter in the remimazolam treatment arm (3.0-7.3 min) than in the midazolam (7.0-15.8 $\mathrm{min})$ or placebo control arms (5.3-21.0 min). Hypotension was observed less frequently in the remimazolam arm in the larger colonoscopy study (Rex et al., 2018); otherwise, the safety profiles of the remimazolam and midazolam arms of these studies were comparable. The mean total dose of remimazolam used to complete the procedure in these studies ranged from $9.0-11.5 \mathrm{mg}$.

The tosylate phase III studies were conducted in colonoscopy (Chen et al., 2020a) and upper gastrointestinal endoscopy (Chen et al., 2021). Propofol was used as the comparator in these studies with a non-inferiority design. The primary endpoints were met in both studies with high sedation success rates for remimazolam that were statistically non-inferior (colonoscopy $=96.9 \%$, upper gastrointestinal endoscopy $=97.3 \%$ ) but numerically lower than those for propofol (100\% in both studies). A slightly slower onset time was observed in the colonoscopy study after remimazolam treatment $(100 \mathrm{~s})$ than propofol treatment $(75 \mathrm{~s})$ with no difference in offset time. In the upper gastrointestinal study, patients treated with remimazolam also showed a slower onset time than propofol but had a faster offset time (time to fully alert, remimazolam $5.75 \mathrm{~min}$, propofol $6.71 \mathrm{~min}$ ). Hypotension was observed in fewer patients in the remimazolam arms of these studies (colonoscopy 23.7\%; upper G.I. endoscopy 13.0\%) than the propofol arms (colonoscopy 51.1\%; upper G.I. endoscopy $42.9 \%)$. Respiratory depression was also significantly lower in the remimazolam arms of these studies (colonoscopy 3.1\%; upper G.I. endoscopy $1.1 \%$ ) than the propofol arms (colonoscopy 16.9\%; upper G.I. endoscopy 6.9\%). Mild gait disorders and dizziness were reported with a similar incidence in the two arms of the colonoscopy study. Injection site pain was only observed for one subject in the remimazolam arms in the two studies combined compared with $10-16 \%$ of the propofol treatment arms.

\section{General Anesthesia}

Benzodiazepines have been employed to provide anesthesia's hypnotic component, but they are not widely used because of the variability of effect, extended duration of action, and lack of control of anesthesia depth (Gamble et al., 1981; Saari et al., 2011). With the observation of the controllable short-acting effects of remimazolam, it became clear that it may be a useful agent in anesthesia (for comment see (Masui, 2020; Sneyd and Rigby-Jones, 2020)).

Reports of four clinical trials of the use of remimazolam for total intravenous anesthesia (TIVA) have been published to date (Table 4), two as full papers reporting a phase IIb/III trial and a phase III trial (Doi et al., 2020a; Doi et al., 2020b), and two reporting phase II trials, one as a short report and abstract (Doi, 2014; Doi et al., 2015) and one as a series of abstracts (Probst et al., 2014, 2015; Bevilacqua et al., 2015). All are trials with remimazolam besylate and employ continuous infusions of remimazolam, co-administered with an opiate analgesic (fentanyl or remifentanil) and neuromuscular blockade, usually with rocuronium. In contrast to procedural sedation, doses corrected for body weight were employed for this indication. Data from the phase II studies are relatively sparse, but successful anesthesia, as defined by no requirement for rescue medication, is reported in both trials after induction and maintenance infusion of remimazolam (Doi, 2014; Probst et al., 2014). Rapid onset of effect was observed as well as a rapid recovery after the surgery. There were no signs of awakening during the surgery nor any memory of the procedure (Doi et al., 2015). A reduced requirement for vasopressor treatment compared with propofol/sevoflurane was observed in the cardiac surgery study (Probst et al., 2015).

Significantly more detailed information is available for the phase II/III and III studies. For induction of anesthesia, a short infusion of 6 or $12 \mathrm{mg} / \mathrm{kg} / \mathrm{h}$ was used, and for maintenance of anesthesia, a dose of $1-2 \mathrm{mg} / \mathrm{kg} / \mathrm{h}$. The primary endpoints were successful anesthesia as defined by the lack of intra-operative awakening or recall, no requirement for rescue sedation, and no 
body movement. The primary endpoints were met with successful anesthesia achieved for all subjects in both studies. Time to loss of consciousness was slightly longer for remimazolam (102 s and $88.7 \mathrm{~s}$ for $6 \mathrm{mg} / \mathrm{kg} / \mathrm{h}$ and $12 \mathrm{mg} / \mathrm{kg} / \mathrm{h}$ induction doses, respectively) when compared to propofol (78.7 s) (Doi, et al., 2020a). Time from ceasing the infusion of the drug to extubation was also slightly longer in the remimazolam arms (19.2 $\mathrm{min})$ compared to propofol (13.1 min) (Doi, et al., 2020a). There was a lower incidence of hypotension in subjects receiving remimazolam (22\%) than those receiving propofol (49.3\%). Fewer patients treated with remimazolam required vasopressors $(41.3 \%)$ or treatment for bradycardia (6.3\%) compared with the propofol arm $(64.0$ and $9.3 \%$, respectively). Pain on injection was reported for $18.7 \%$ of subjects treated with propofol but none treated with remimazolam. The only adverse drug reaction that was higher in the remimazolam arms vs. propofol arms was nausea and vomiting ( 7 and $6 \%$ respectively for remimazolam and 5.3 and $4 \%$ for propofol). One of the studies examined clinically vulnerable patients (ASA Class III). Efficacy and safety data were similar to those in ASA I and II patients (Doi, et al., 2020b).

The mean length of procedure in the large phase II/III study was $\sim 150 \mathrm{~min}$, and the mean total dose of remimazolam was $3.47 \mathrm{mg} / \mathrm{kg}$ (Doi et al., 2020a). This translates to $\sim 240 \mathrm{mg}$ for a $70 \mathrm{~kg}$ individual, compared with $\sim 10 \mathrm{mg}$ for the procedural sedation studies (Procedural sedation).

\section{Case Reports}

Since reaching the market, several case reports have been published on the use of remimazolam (Table 5). The use in awake craniotomy is of particular interest, especially incorporating the planned use of flumazenil to reverse sedation during the procedure (Yoshida et al., 2021). However, Tachibana et al. (Tachibana et al., 2021) have questioned the effectiveness of remimazolam for craniotomy based on observation from two subjects. They make three points. Firstly, that increased sputum is produced compared to propofol due to the lower suppression of the pharyngeal reflex by remimazolam; secondly, that, because remimazolam has a lower effect on blood pressure compared to propofol, surgical difficulties can result due to higher intracranial pressure and thirdly that agitation was observed in one subject upon flumazenil reversal. Clearly, further study is required on the use of remimazolam in craniotomy to understand better the risk/ benefit balance.

Successful use of remimazolam has been described in spine surgery incorporating motor evoked potential monitoring (Kondo et al., 2020), thyroid surgery with neuromonitoring (Hayamizu et al., 2021), and cardiac surgery with cardiopulmonary bypass (Saito et al., 2021). Remimazolam's successful use in high-risk patients, including endoscopic retrograde cholangiopancreatography in a patient with impaired respiratory function due to myotonic dystrophy (Fukuda et al., 2021), MitraClip implantation in a patient with advanced heart failure (Satoh et al., 2021), and cochlear implant in a patient with mitochondrial myopathy (Suzuki et al., 2021) has been published.
In the procedural sedation setting, Henson and Thompson (Henson and Thompson, 2021) report that sedation with remimazolam resulted in a lower "insult" to neuropsychiatric function in geriatric patients undergoing a variety of procedures compared to existing agents.

There has been an observation of re-sedation after flumazenil reversal (Yamamoto et al., 2021b; Yamamoto et al., 2021a). This may have been due to residual remimazolam, the metabolite CNS 7054 building up to levels that can activate the $\mathrm{GABA}_{\mathrm{A}}$ receptor or a combination of both, potentially as a result of compromised organ function. There are also reports of precipitation of remimazolam in Ringer's solution (Yoshida et al., 2021; Matsuo et al., 2021; Sasaki et al., 2021). A single report of anaphylaxis resulting from the administration of remimazolam has been published (Tsurumi et al., 2021).

Further case reports that shed light on the utility of remimazolam in practice can be expected in the coming years.

\section{Adverse Reactions and Liabilities}

The two salts of remimazolam have very similar adverse reaction profiles, which are in line with those observed with other classical benzodiazepines. The most frequently observed adverse reactions in the procedural sedation setting are changes in blood pressure and heart rate, reduced respiratory rate, and vomiting. The incidence of these events was comparable with midazolam comparator groups (Rex et al., 2018; Pastis et al., 2019). In high-risk patients (ASA class III/IV), a safety profile similar to that in low-risk patients was observed (Rex et al., 2021). Compared with propofol in procedural sedation, remimazolam showed a lower incidence of hypotension, respiratory depression, and injection site pain (Chen et al., 2020a; Chen et al., 2021; Zhang et al., 2021).

The most common adverse reactions in anesthesia are hypotension, vomiting, and nausea, (Doi, et al., 2020b; Doi, et al., 2020a). The safety profile of remimazolam in high-risk patients (ASA class III) was in line with corresponding results from ASA class I and II patients (Doi, et al., 2020b). In comparison to propofol, the incidence of hypotension, requirement to treat for bradycardia, and injection site pain were lower in the remimazolam arms, while propofol may be slightly superior with respect to postoperative nausea and vomiting (PONV) (Doi, et al., 2020a). There was also a trend toward reduced nausea and vomiting in the propofol arm of the phase III colonoscopy study compared with remimazolam tosylate (Chen et al., 2020a). Both propofol and midazolam are recognized to have anti-emetic properties (Soppitt et al., 2000; Ahn et al., 2016). Further studies to evaluate the incidence of PONV after remimazolam treatment, in comparison to propofol, would be of interest.

Benzodiazepines are known to induce tolerance and dependence on repeated use and to have abuse potential (O'brien, 2005). Remimazolam is likely to have the same liabilities, but they are probably mitigated to an extent because of the short duration of effect and the requirement for intravenous administration. Indeed, Io et al. (Io et al., 2021) observed tolerance to the effects of remimazolam over a 28 days dosing period in the pig, but note that the tolerance observed 


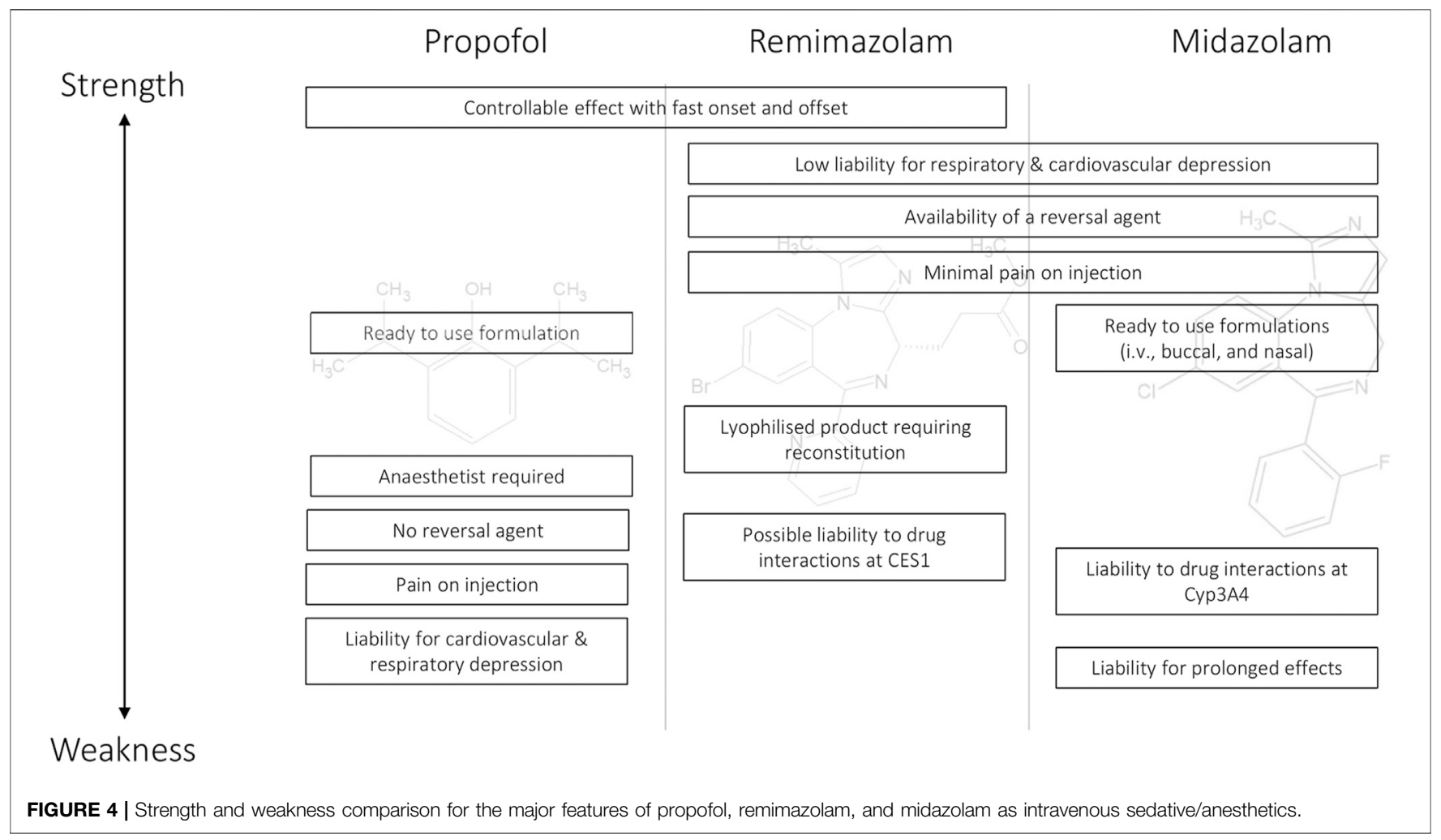

(assessed by the requirement to increase the dose to maintain the same sedative effect) was less than that seen with midazolam. As might be predicted, a reduced sedative effect of remimazolam has been observed in subjects with an existing tolerance to benzodiazepines resulting from long-term use equivalent to 15-20 mg diazepam per day (Yoshikawa et al., 2021).

In a comparative study of recreational drug users, it was concluded that remimazolam does have the potential for abuse but that this was comparable to or lower than that of midazolam (Schippers et al., 2020). The authors note midazolam itself has a low potential for intravenous abuse. Pesic et al. (Pesic et al., 2020a) conducted a trial in female subjects and report that remimazolam besylate does not have the potential for misuse to facilitate sexual assaults because of its very low oral bioavailability and bitter taste.

Synergistic pharmacological interaction between benzodiazepines and opioid analgesics is well established. This is usually beneficial in both procedural sedation and anesthesia settings. The synergy with opioid analgesics has been clearly established for remimazolam (Bevans et al., 2017; Zhou et al., 2020a; Kops et al., 2021; Zhou et al., 2021).

Remimazolam is thought to have a lower potential for pharmacokinetic drug interactions than midazolam. Midazolam is metabolized primarily by cytochrome P450 3A4, a common drug metabolism enzyme that is inhibited by several drugs leading to interaction issues (Yuan et al., 1999). One factor to consider, however, is that the reported major metabolizing enzyme of remimazolam, CES1, is inhibited by naturally occurring agents such as flavonoids and fatty acids (Crow et al., 2010; Wang et al., 2018). Alcohol, an inhibitor of CES1, has been shown to increase exposure to remimazolam (Pesic et al., 2020a).

\section{Alternative Indications and Routes of Administration}

The properties of remimazolam potentially make it a useful agent for ICU sedation, especially to facilitate light sedation protocols and rapid awakening (Barr et al., 2013). The original Japanese licensee for remimazolam besylate, Ono Pharmaceutical Co., conducted a study in ICU sedation. No peer-reviewed paper report of this study appears to be available; however, Zhou et al. (Zhou et al., 2020a) note that "data from 49 Japanese patients in the intensive care unit (ICU) showed that seven subjects treated longer than $24 \mathrm{~h}$ had higher than expected concentrations of remimazolam for samples collected $>24 \mathrm{~h}$ after the initiation of remimazolam dosing." Further evaluation of the extended effects observed is warranted, as is a further study of the potential utility of remimazolam in ICU sedation. Note that remimazolam has already been approved in Belgium for compassionate use in ICU sedation (FAMHP, 2020), owing to a shortage of current medications resulting from the COVID-19 crisis.

Remimazolam is unlikely to find use by the oral route because of its very low bioavailability $(\sim 1-2 \%)$, which is largely a result of extensive first-pass metabolism (Pesic et al., 2020a). Bioavailability by the intranasal route is significantly higher $(\sim 50 \%)$, and sedative effects were observed after administration to human volunteers via this route. The 
formulations that were employed in this study (powder and the intravenous formulation of remimazolam besylate), however, caused significant nasal discomfort/pain (Pesic et al., 2020b). Otherwise, the drug was reported to be safe and well-tolerated by this route. If a more tolerable formulation can be developed, intranasal delivery for settings where intravenous administration is problematic such as in epilepsy, agitation or for pediatric sedative use (Fantacci et al., 2018; Shioji et al., 2021), may be feasible. Inhalation has also been proposed as an alternative delivery route, and some animal data supports this, showing that inhaled remimazolam potentiates the effects of inhaled remifentanil (Bevans et al., 2017).

\section{Flumazenil Reversal}

Flumazenil is an antagonist to the positive allosteric modulator effects of benzodiazepines at the $\mathrm{GABA}_{\mathrm{A}}$ receptor (Hunkeler et al., 1981). The compound is approved for clinical use for reversal of benzodiazepine effects, usually resulting from overdose. Reversal or prevention of the effects of remimazolam by flumazenil has been clearly demonstrated in pre-clinical (Kilpatrick et al., 2007) and clinical studies (Worthington et al., 2013; Chen et al., 2020b). In the anesthesia phase III studies, flumazenil was used as a reversal agent in subjects $(\sim 5-10 \%)$ who had not awoken within $30 \mathrm{~min}$ of the termination of remimazolam infusion (Doi, et al., 2020b; Doi, et al., 2020a). The availability of a reversal agent provides a safety feature in case of overdose or adverse events that is not available for many other anesthetics, including propofol (Sneyd and Rigby-Jones, 2020). An important factor to consider, however, is that flumazenil is itself an ester-based drug and has a short half-life and duration of action (Lauven et al., 1985), so multiple doses may be required to avoid re-sedation events.

The planned use of flumazenil in combination with remimazolam to control sedation levels precisely may open new avenues in procedural sedation and anesthesia. The use of flumazenil to reverse remimazolam-induced anesthesia during a craniotomy procedure to awaken the patient is an interesting example (Yoshida et al., 2021).

\section{CONCLUSION AND FUTURE DIRECTIONS}

The aim of this review is to provide an analysis of, and perspective on, published non-clinical and clinical information on remimazolam. The recent introduction of this compound to the market appears to provide a valuable addition to the armory of agents available for procedural sedation and anesthesia. Remimazolam has the pharmacological profile of a classical benzodiazepine but is differentiated by its ester group and rapid metabolism, via tissue esterases, to a significantly less active metabolite. The compound exhibits rapid pharmacokinetics and pharmacodynamics with relatively small effects of covariates such as age, gender, race, obesity status, ASA class, and weight.

A comparison of the strengths and weaknesses of remimazolam with midazolam and propofol is shown in
Figure 4. The efficacy profile of remimazolam with a predictable and controllable sedative effect and fast onset and offset improves on midazolam. The safety profile of limited cardiovascular and respiratory depression, minimal pain on injection, and the availability of a reversal agent offer advantages over propofol. One complicating feature with the use of remimazolam is that it is a lyophilized product requiring reconstitution rather than the ready-to-use formulations available for propofol and midazolam. The availability of remimazolam is likely to change routine clinical practice in the procedural sedation setting because its efficacy/safety profile will facilitate routine procedures to be conducted rapidly with low liability for deep and/or prolonged sedation. In the anesthesia setting, the compound has the potential to be especially useful in high-risk patients, given the low impact on the cardiovascular and respiratory systems coupled with the availability of a reversal agent.

Comprehension of the complete profile of remimazolam will increase as its use widens, but gaps exist currently. A better understanding is required of the occasional cases of prolonged sedation. A full in vitro analysis of the metabolism of remimazolam by various human tissues and characterization of metabolism by specific carboxylesterases and their genetic variants would be a useful addition to the literature. This, together with analysis of the liability for interactions with other drugs metabolized by carboxylesterases, would help to understand potential individual variability in its metabolism and sedative/ anesthetic effects. Further clinical data would help to understand better the profile of remimazolam with respect to postoperative complications such as nausea and vomiting. Target engagement analysis employing techniques such as positron emission tomography would provide an enhanced understanding of the interaction of remimazolam with the benzodiazepine site on the $\mathrm{GABA}_{\mathrm{A}}$ receptor and may facilitate improved $\mathrm{PK} / \mathrm{PD}$ modeling.

Following the discovery of the compound and early characterisation of the base, the besylate salt form of remimazolam was taken into development. A second development program of the closely related tosylate salt was subsequently initiated in China. Both salt forms have now reached market authorization. No head-to-head comparisons have yet been conducted, but their clinical profiles appear to be virtually identical, as one might expect, given the minor difference. The development challenges of remimazolam (besylate) are worthy of comment. It is unusual for a small company with limited resources to take a compound to the market. While it has taken considerably longer than the industry average to progress to market, this has been achieved by the developer, its predecessors, and its licensees.

The use of intravenous remimazolam is likely to be extended soon to other settings, including sedation in intensive care. Target controlled infusion techniques, employing models of plasma drug concentrations, could enhance its use in general anesthesia. The development of new formulations, e.g., intranasal or inhalation, would enable additional use in situations where intravenous administration 
is difficult, such as pediatrics, epilepsy, or severe agitation. The availability of this new agent, potentially used in combination with the reversal agent, flumazenil, may also permit further innovations in anesthesia and sedation techniques.

\section{REFERENCES}

Ahn, E. J., Kang, H., Choi, G. J., Baek, C. W., Jung, Y. H., and Woo, Y. C. (2016). The Effectiveness of Midazolam for Preventing Postoperative Nausea and Vomiting. Anesth. Analgesia 122 (3), 664-676. doi:10.1213/ ANE.0000000000001062

Antonik, L. J., Goldwater, D. R., Kilpatrick, G. J., Tilbrook, G. S., and Borkett, K. M. (2012). A Placebo- and Midazolam-Controlled Phase I Single Ascending-Dose Study Evaluating the Safety, Pharmacokinetics, and Pharmacodynamics of Remimazolam (CNS 7056). Anesth. Analgesia 115 (2), 274-283. doi:10.1213/ ANE.0b013e31823f0c28

Barr, J., Fraser, G. L., Puntillo, K., Ely, E. W., Gélinas, C., Dasta, J. F., et al. (2013). Clinical Practice Guidelines for the Management of Pain, Agitation, and Delirium in Adult Patients in the Intensive Care Unit. Crit. Care Med. 41 (1), 263-306. doi:10.1097/CCM.0b013e3182783b72

Bauer, T. M., Ritz, R., Haberthür, C., Haefeli, W. E., Scollo-Lavizzari, G., Ha, H. R., et al. (1995). Prolonged Sedation Due to Accumulation of Conjugated Metabolites of Midazolam. The Lancet 346 (8968), 145-147. doi:10.1016/ s0140-6736(95)91209-6

Bevans, T., Deering-Rice, C., Stockmann, C., Rower, J., Sakata, D., and Reilly, C. (2017). Inhaled Remimazolam Potentiates Inhaled Remifentanil in Rodents. Anesth. Analg. 124 (5), 1484-1490. doi:10.1213/ANE.0000000000002022

Bevilacqua, C., Probst, S., Soehngen, M., and Ender, J. (2015). The Sedative Effect of Remimazolam in General Anesthesia for Cardiac Surgery Measured by Narcotrend. J. Cardiothorac. Vasc. Anesth. 29 (Suppl. 2), S50-S51. doi:10.1053/j.jvca.2015.05.267

Birgenheier, N. M., Stuart, A. R., and Egan, T. D. (2020). Soft Drugs in Anesthesia: Remifentanil as Prototype to Modern Anesthetic Drug Development. Curr. Opin. Anaesthesiol. 33, 499-505. doi:10.1097/ACO.0000000000000879

Borkett, K. M., Riff, D. S., Schwartz, H. I., Winkle, P. J., Pambianco, D. J., Lees, J. P., et al. (2015). A Phase IIa, Randomized, Double-Blind Study of Remimazolam (CNS 7056) versus Midazolam for Sedation in Upper Gastrointestinal Endoscopy. Anesth. Analg. 120 (4), 771-780. doi:10.1213/ANE.0000000000000548

Bower, A. L., Ripepi, A., Dilger, J., Boparai, N., Brody, F. J., and Ponsky, J. L. (2000). Bispectral index Monitoring of Sedation during Endoscopy. Gastrointest. Endosc. 52 (2), 192-196. doi:10.1067/mge.2000.107284

Bray, R. J. (1998). Propofol Infusion Syndrome in Children. Pediatr. Anesth. 8 (6), 491-499. doi:10.1046/j.1460-9592.1998.00282.x

Carithers, L. J., Ardlie, K., Barcus, M., Branton, P. A., Britton, A., Buia, S. A., et al. (2015). A Novel Approach to High-Quality Postmortem Tissue Procurement: The GTEx Project. Biopreservation and Biobanking 13 (5), 311-319. doi:10.1089/bio.2015.0032

CES1 gene expression (2021a). GTEX Gene Exptression Database, V8. Available at: https://www.gtexportal.org/home/gene/CES1.

CES1 protein expression (2021b). Human Protein Atlas Database. Available at: https://www.proteinatlas.org/ENSG00000198848-CES1/tissue.

Chen, S., Wang, J., Xu, X., Huang, Y., Xue, S., Wu, A., et al. (2020a). The Efficacy and Safety of Remimazolam Tosylate versus Propofol in Patients Undergoing Colonoscopy: a Multicentered, Randomized, Positive-Controlled, Phase III Clinical Trial. Am. J. Transl Res. 12 (8), 4594-4603.

Chen, S. H., Yuan, T. M., Zhang, J., Bai, H., Tian, M., Pan, C. X., et al. (2021). Remimazolam Tosilate in Upper Gastrointestinal Endoscopy: A Multicenter, Randomized, Non-inferiority, Phase III Trial. J. Gastroenterol. Hepatol. 36 (2), 474-481. doi:10.1111/jgh.15188

Chen, X., Sang, N., Song, K., Zhong, W., Wang, H., Jiang, J., et al. (2020b). Psychomotor Recovery Following Remimazolam-Induced Sedation and the Effectiveness of Flumazenil as an Antidote. Clin. Ther. 42 (4), 614-624. doi:10.1016/j.clinthera.2020.02.006

Chernik, D. A., Gillings, D., Laine, H., Hendler, J., Silver, J. M., Davidson, A. B., et al. (1990). Validity and Reliability of the Observer??s. J. Clin. Psychopharmacol. 10 (4), 244-251. doi:10.1097/00004714-199008000-00003

\section{AUTHOR CONTRIBUTIONS}

The author confirms being the sole contributor of this work and has approved it for publication.

Cornett, E. M., Novitch, M. B., Brunk, A. J., Davidson, K. S., Menard, B. L., Urman, R. D., et al. (2018). New Benzodiazepines for Sedation. Best Pract. Res. Clin. Anaesthesiol. 32, 149-164. doi:10.1016/j.bpa.2018.06.007

Cornett, P. M., Matta, J. A., and Ahern, G. P. (2008). General Anesthetics Sensitize the Capsaicin Receptor Transient Receptor Potential V1. Mol. Pharmacol. 74 (5), 1261-1268. doi:10.1124/mol.108.049684

Crow, J. A., Herring, K. L., Xie, S., Borazjani, A., Potter, P. M., and Ross, M. K. (2010). Inhibition of Carboxylesterase Activity of THP1 Monocytes/ macrophages and Recombinant Human Carboxylesterase 1 by Oxysterols and Fatty Acids. Biochim. Biophys. Acta (Bba) - Mol. Cel Biol. Lipids 1801 (1), 31-41. doi:10.1016/j.bbalip.2009.09.002

Doi, M., Hirata, N., Suzuki, T., Morisaki, H., Morimatsu, H., and Sakamoto, A. (2020b). Safety and Efficacy of Remimazolam in Induction and Maintenance of General Anesthesia in High-Risk Surgical Patients (ASA Class III): Results of a Multicenter, Randomized, Double-Blind, Parallel-Group Comparative Trial. J. Anesth. 34 (4), 491-501. doi:10.1007/s00540-020-02776-w

Doi, M., Morita, K., Shiraishi, Y., Katoh, T., Kurita, T., Igarashi, H., et al. (2015). Remimazolam Dose Finding Studies for Anesthetic/Sedative in the IndicationGeneral Anesthesia in Japanese Volunteers/Patients. Anesthesiology, A3011.

Doi, M., Morita, K., Takeda, J., Sakamoto, A., Yamakage, M., and Suzuki, T. (2020a). Efficacy and Safety of Remimazolam versus Propofol for General Anesthesia: a Multicenter, Single-Blind, Randomized, Parallel-Group, Phase IIb/III Trial. J. Anesth. 34 (4), 543-553. doi:10.1007/s00540-020-02788-6

Doi, M. (2014). Remimazolam. Jjsca. 34, 860-866. doi:10.2199/jjsca.34.860

Dossa, F., Megetto, O., Yakubu, M., Zhang, D. D. Q., and Baxter, N. N. (2021). Sedation Practices for Routine Gastrointestinal Endoscopy: a Systematic Review of Recommendations. BMC Gastroenterol. 21 (1), 22. doi:10.1186/s12876-02001561-z

Dundee, J. W., Halliday, N. J., Harper, K. W., and Brogden, R. N. (1984). Midazolam A Review of its Pharmacological Properties and Therapeutic Use. Drugs 28 (6), 519-543. doi:10.2165/00003495-198428060-00002

Egan, T. D., Obara, S., Jenkins, T. E., Jaw-Tsai, S. S., Amagasu, S., Cook, D. R., et al. (2012). Azd-3043. Anesthesiology 116 (6), 1267-1277. doi:10.1097/ ALN.0b013e31825685a6

Eisenried, A., Schüttler, J., Lerch, M., Ihmsen, H., and Jeleazcov, C. (2020). Pharmacokinetics and Pharmacodynamics of Remimazolam (CNS 7056) after Continuous Infusion in Healthy Male Volunteers. Anesthesiology. 132, 652-666. doi:10.1097/ALN.0000000000003102

EMA (2021). 'EMA', European Medicines Agency. Available at: https://www.ema. europa.eu/en/medicines/human/paediatric-investigation-plans/emea-001880pip02-19.

Erhardt, P. W., Woo, C. M., Anderson, W. G., and Gorczynski, R. J. (1982). Ultrashort-acting .beta.-adrenergic Receptor Blocking Agents. 2. (Aryloxy) propanolamines Containing Esters on the Aryl Function. J. Med. Chem. 25 (12), 1408-1412. doi:10.1021/jm00354a003

FAMHP (2020). 'FAMHP', Products, Federal Agency for Medicines And Health. Available at: https://www.famhp.be/en/human_use/medicines/medicines/ research_development/compassionate_use_medical_need.

Fantacci, C., Fabrizio, G. C., Ferrara, P., Franceschi, F., and Chiaretti, A. (2018). Intranasal Drug Administration for Procedural Sedation in Children Admitted to Pediatric Emergency Room. Eur. Rev. Med. Pharmacol. Sci. 22 (1), 217-222. doi:10.26355/eurrev_201801_14120

FDA (2020a). Byfavo: Highlights of Prescribing Information. Available at: https:// www.accessdata.fda.gov/drugsatfda_docs/label/2020/212295s000lbl.pdf.

FDA (2020b). 'Drug Approval Package: BYFAVO', FDA Website. Available at: https://www.accessdata.fda.gov/drugsatfda_docs/nda/2020/212295Orig1s000TOC.cfm.

Feldman, P. L. (2020). Insights into the Chemical Discovery of Remifentanil. Anesthesiology. United States 132 (5), 1229-1234. doi:10.1097/ ALN.0000000000003170

Feldman, P. L., James, M. K., Brackeen, M. F., Bilotta, J. M., Schuster, S. V., Lahey, A. P., et al. (1991). Design, Synthesis, and Pharmacological Evaluation of 
Ultrashort- to Long-Acting Opioid Analgesics. J. Med. Chem.United States 34 (7), 2202-2208. doi:10.1021/jm00111a041

Freyer, N., Knöspel, F., Damm, G., Greuel, S., Schneider, C., Seehofer, D., et al. (2019). Metabolism of Remimazolam in Primary Human Hepatocytes during Continuous Long-Term Infusion in a 3-D Bioreactor System. Drug Des. Devel Ther. 13, 1033-1047. doi:10.2147/ DDDT.S186759

Fukuda, M., Tachibana, S., Nishihara, N., and Yamakage, M. (2021). Remimazolam for a Patient with Myotonic Dystrophy Type 1 Who Underwent Endoscopic Retrograde Cholangiopancreatography under General Anesthesia: a Case Report. JA Clin. Rep. 7 (1), 17. doi:10.1186/s40981-021-00422-2

Gabriele, M., Puccini, P., Lucchi, M., Vizziello, A., Gervasi, P. G., and Longo, V. (2018). Presence and Inter-individual Variability of Carboxylesterases (CES1 and CES2) in Human Lung. Biochem. Pharmacol. 150, 64-71. doi:10.1016/ j.bcp.2018.01.028

Gamble, J. A. S., Kawar, P., Dundee, J. W., Moore, J., and Briggs, L. P. (1981). Evaluation of Midazolam as an Intravenous Induction Agent. Anaesthesia 36 (9), 868-873. doi:10.1111/j.1365-2044.1981.tb08859.x

Ge, R., Pejo, E., Husain, S. S., Cotten, J. F., and Raines, D. E. (2012). Electroencephalographic and Hypnotic Recoveries after Brief and Prolonged Infusions of Etomidate and Optimized Soft Etomidate Analogs. Anesthesiology 117 (5), 1037-1043. doi:10.1097/ ALN.0b013e31826d3de2

Glen, J. B., and Hunter, S. C. (1984). Pharmacology of an Emulsion Formulation of ICI 35 868. Br. J. Anaesth. 56 (6), 617-626. doi:10.1093/bja/56.6.617

Gründer, G., Siessmeier, T., Lange-Asschenfeldt, C., Vernaleken, I., Buchholz, H.G., Stoeter, P., et al. (2001). [ 18 F]Fluoroethylflumazenil: a Novel Tracer for PET Imaging of Human Benzodiazepine Receptors. Eur. J. Nucl. Med. Mol. Imaging 28 (10), 1463-1470. doi:10.1007/s002590100594

Haefely, W. E., Martin, J. R., Richards, J. G., and Schoch, P. (1993). The Multiplicity of Actions of Benzodiazepine Receptor Ligands. Can. J. Psychiatry 38 Suppl 4 (Suppl. 4), S102-S108.

Hayamizu, K., Chaki, T., Tachibana, S., Hirata, N., and Yamakage, M. (2021). Effect of Remimazolam on Intraoperative Neuromonitoring during Thyroid Surgery: a Case Series. J. Anesth. doi:10.1007/s00540-021-02955-3

Hegarty, J. E., and Dundee, J. W. (1977). Sequelae after the Intravenous Injection of Three Benzodiazepines-Ddiazepam, Lorazepam, and Flunitrazepam. Bmj 2 (6099), 1384-1385. doi:10.1136/bmj.2.6099.1384

Hemphill, S., McMenamin, L., Bellamy, M. C., and Hopkins, P. M. (2019). Propofol Infusion Syndrome: a Structured Literature Review and Analysis of Published Case Reports. Br. J. Anaesth. 122 (4), 448-459. doi:10.1016/ j.bja.2018.12.025

Henson, K. S., and Thompson, K. J. (2021). What May Be the First Phase 4 Experience with BYFAVO (Remimazolam). AANA J. 89, 101-102.

Hirata, N., Hayamizu, K., and Yamakage, M. (2020). How to Administer Remimazolam for Anesthesia Induction. J. Anesth. 34, 962. doi:10.1007/ s00540-020-02864-x

Hu, K., Xiang, Q., Wang, Z., Sheng, X., Li, L. e., Liang, Y., et al. (2021). Effects of Vitamin D Receptor, Cytochrome P450 3A, and Cytochrome P450 Oxidoreductase Genetic Polymorphisms on the Pharmacokinetics of Remimazolam in Healthy Chinese Volunteers. Clin. Pharmacol. Drug Develop. 10 (1), 22-29. doi:10.1002/cpdd.797

Hunkeler, W., Möhler, H., Pieri, L., Polc, P., Bonetti, E. P., Cumin, R., et al. (1981). Selective Antagonists of Benzodiazepines. Nature 290 (5806), 514-516. doi:10.1038/290514a0

International Nonproprietary Names for Pharmaceutical Substances (INN) (2010) WHO Drug Information, 24(3), p. 277.

Iguchi, S., Iwamura, H., Nishizaki, M., Hayashi, A., Senokuchi, K., Kobayashi, K., et al. (1992). Development of a Highly Cardioselective Ultra Short-Acting .BETA.-Blocker, ONO-1101. Chem. Pharm. Bull. 40 (6), 1462-1469. doi:10.1248/cpb.40.1462

Io, T., Saunders, R., Pesic, M., Petersen, K.-U., and Stoehr, T. (2021). A Miniature Pig Model of Pharmacological Tolerance to Long-Term Sedation with the Intravenous Benzodiazepines; Midazolam and Remimazolam. Eur. J. Pharmacol. 896, 173886. doi:10.1016/j.ejphar.2021.173886

Johnson, K. B. (2012). New Horizons in Sedative Hypnotic Drug Development. Anesth. Analgesia 115, 220-222. doi:10.1213/ ANE.0b013e31825ef8d7
Jurd, R., Arrasa, M., Lambert, S., Drexler, B., Siegwart, R., Crestani, F., et al. (2003). General Anesthetic Actionsin Vivostrongly Attenuated by a point Mutation in the GABAAreceptor $\beta 3$ Subunit. FASEB j. 17 (2), 250-252. doi:10.1096/fj.020611fje

Kamal, F., Khan, M. A., Lee-Smith, W., Sharma, S., Imam, Z., Jowhar, D., et al. (2021). Efficacy and Safety of Supplemental Intravenous Lidocaine for Sedation in Gastrointestinal Endoscopic Procedures: Systematic Review and MetaAnalysis of Randomized Controlled Trials. Gastrointest. Endosc. 93, 1241-1249. doi:10.1016/j.gie.2021.01.008

Kanto, J. H. (1985). Midazolam: The First Water-Soluble Benzodiazepine; Pharmacology, Pharmacokinetics and Efficacy in Insomnia and Anesthesia. United States 5 (3), 138-155. doi:10.1002/j.1875-9114.1985.tb03411.x

Keam, S. J. (2020). Remimazolam: First Approval. Drugs 80, 625-633. doi:10.1007/ s40265-020-01299-8

Kilpatrick, G. J., McIntyre, M. S., Cox, R. F., Stafford, J. A., Pacofsky, G. J., Lovell, G. G., et al. (2007). CNS 7056: A Novel Ultra-short-acting Benzodiazepine. Anesthesiology 107 (1), 60-66. doi:10.1097/01.anes.0000267503.85085.c0

Kilpatrick, G. J., and Tilbrook, G. S. (2006). Drug Development in Anaesthesia: Industrial Perspective. Curr. Opin. Anaesthesiology 19 (4), 385-389. doi:10.1097/01.aco.0000236137.23475.95

Kilpatrick, G. J., McIntyre, M. S., Cox, R. F., Stafford, J. A., and Pacofsky, G. J. (2006a). CNS 7056X, an Ultra-Short Acting Benzodiazepine: In Vitro Ligand Binding Profile. Anesthesiology 105, A543.

Kilpatrick, G. J., Wiard, R. P., Stafford, J. A., Feldman, P. L., and Tilbrook, G. S. (2006b). CNS 7056X, an Ultra-short Acting Benzodiazepine: Sedation Profile in theRodent. Anesthesiology 105, A1609.

Kim, J. J., and Hibbs, R. E. (2021). Direct Structural Insights into GABAA Receptor Pharmacology. Trends Biochem. Sci. 46, 502-517. doi:10.1016/ j.tibs.2021.01.011

Kleiman, R. B., Darpo, B., Thorn, M., Stoehr, T., and Schippers, F. (2020). Potential Strategy for Assessing QT/QTc Interval for Drugs that Produce Rapid Changes in Heart Rate: Electrocardiographic Assessment of the Effects of Intravenous Remimazolam on Cardiac Repolarization. Br. J. Clin. Pharmacol. 86 (8), 1600-1609. doi:10.1111/bcp.14270

Kondo, T., Toyota, Y., Narasaki, S., Watanabe, T., Miyoshi, H., Saeki, N., et al. (2020). Intraoperative Responses of Motor Evoked Potentials to the Novel Intravenous Anesthetic Remimazolam during Spine Surgery: a Report of Two Cases. JA Clin. Rep. 6 (1), 97. doi:10.1186/s40981-020-00401-z

Kops, M. S., Pesic, M., Petersen, K.-U., Schmalix, W. A., and Stöhr, T. (2021). Impact of Concurrent Remifentanil on the Sedative Effects of Remimazolam, Midazolam and Propofol in Cynomolgus Monkeys. Eur. J. Pharmacol. 890, 173639. doi:10.1016/j.ejphar.2020.173639

Laizure, S. C., Herring, V., Hu, Z., Witbrodt, K., and Parker, R. B. (2013). The Role of Human Carboxylesterases in Drug Metabolism: Have We Overlooked Their Importance?. Pharmacotherapy 33 (2), 210-222. doi:10.1002/phar.1194

Lauven, P. M., Schwilden, H., Stoeckel, H., and Greenblatt, D. J. (1985). The Effects of a Benzodiazepine Antagonist Ro 15-1788 in the Presence of Stable Concentrations of Midazolam. Anesthesiology. United States 63 (1), 61-64. doi:10.1097/00000542-198507000-00009

Liu, Y., Xu, X., Xie, J., Ma, H., Wang, T., Zhang, G., et al. (2016). Design, Synthesis, and Biological Evaluation of Novel CNS 7056 Derivatives as Sedatives in Rats and Rabbits. Chem. Biol. Drug Des. 88 (1), 38-42. doi:10.1111/cbdd.12731

Lohmer, L. L., Schippers, F., Petersen, K. U., Stoehr, T., and Schmith, V. D. (2020). Time-to-Event Modeling for Remimazolam for the Indication of Induction and Maintenance of General Anesthesia. J. Clin. Pharmacol. 60 (4), 505-514. doi: $10.1002 /$ jcph.1552

Makkad, B. (2013). Procedural Sedation for Interventional Cardiology Procedures. Int. anesthesiology Clin. United States 51 (2), 112-126. doi:10.1097/ AIA.0b013e31828ebcfc

Masui, K. (2020). Remimazolam Besilate, a Benzodiazepine, Has Been Approved for General Anesthesia!!. J. Anesth. 34, 479-482. doi:10.1007/s00540-02002755-1

Matsuo, M., Okada, K., Onuki, Y., and Yamazaki, M. (2021). Incompatibility of Remimazolam Besylate with Ringer's Acetate Infusion Resulting in Total Occlusion of an Intravenous Catheter. BMJ Case Rep. 14 (4), e241622. doi:10.1136/bcr-2021-241622

Matta, J. A., Cornett, P. M., Miyares, R. L., Abe, K., Sahibzada, N., and Ahern, G. P. (2008). General Anesthetics Activate a Nociceptive Ion Channel to Enhance 
Pain and Inflammation. Proc. Natl. Acad. Sci. 105 (25), 8784-8789. doi:10.1073/ pnas.0711038105

McKernan, R. M., Rosahl, T. W., Reynolds, D. S., Sur, C., Wafford, K. A., Atack, J. R., et al. (2000). Sedative but Not Anxiolytic Properties of Benzodiazepines Are Mediated by the GABAA Receptor al Subtype. Nat. Neurosci. 3 (6), 587-592. doi:10.1038/75761

Mundipharma Co. Ltd (2020). Remimazolam Besylate Anerem for Intravenous Injection 50mg. Preclinical Study Summary and Summary Table. Available at: https://www.pmda.go.jp/drugs/2020/P20200120002/770098000_30200AMX00031_ H100_1.pdf.

Mutter, C., Rudolf, G., Diemunsch, P. A., Tilbrook, G. S., and Borgeat, A. (2006). CNS 7056X an Ultra-short Acting Benzodiazepine: Pharmacokinetic andPharmacodynamic Study in Pig. Anesthesiology 105, A1610.

O'brien, C. P. (2005). Benzodiazepine Use, Abuse, and Dependence. J. Clin. Psychiatry United States 66 (Suppl. 2), 28-33.

Pacofsky, G. J., Stafford, J. A., Cox, R. F., Cowan, J. R., Dorsey, G. F., Gonzales, S. S., et al. (2002). Relating the Structure, Activity, and Physical Properties of Ultrashort-Acting Benzodiazepine Receptor Agonists. Bioorg. Med. Chem. LettersEngland 12 (21), 3219-3222. doi:10.1016/s0960-894x(02)00513-9

Pambianco, D. J., Borkett, K. M., Riff, D. S., Winkle, P. J., Schwartz, H. I., Melson, T. I., et al. (2016). A Phase IIb Study Comparing the Safety and Efficacy of Remimazolam and Midazolam in Patients Undergoing Colonoscopy. Gastrointest. Endosc. 83 (5), 984-992. doi:10.1016/j.gie.2015.08.062

Parker, R. B., Hu, Z.-Y., Meibohm, B., and Laizure, S. C. (2015). Effects of Alcohol on Human Carboxylesterase Drug Metabolism. Clin. Pharmacokinet. 54 (6), 627-638. doi:10.1007/s40262-014-0226-2

Pastis, N. J., Yarmus, L. B., Schippers, F., Ostroff, R., Chen, A., Akulian, J., et al. (2019). Safety and Efficacy of Remimazolam Compared with Placebo and Midazolam for Moderate Sedation during Bronchoscopy. Chest 155 (1), 137-146. doi:10.1016/j.chest.2018.09.015

Perel, A. (2011). Non-anaesthesiologists Should Not Be Allowed to Administer Propofol for Procedural Sedation. Eur. J. Anaesthesiology 28 (8), 580-584. doi:10.1097/EJA.0b013e328348a977

Persson, A., Ehrin, E., Eriksson, L., Farde, L., Hedström, C.-G., Litton, J.-E., et al. (1985). Imaging of [11C]-Labelled Ro 15-1788 Binding to Benzodiazepine Receptors in the Human Brain by Positron Emission Tomography. J. Psychiatr. Res. 19 (4), 609-622. doi:10.1016/0022-3956(85)90080-9

Pesic, M., Schippers, F., Saunders, R., Webster, L., Donsbach, M., and Stoehr, T. (2020a). Pharmacokinetics and Pharmacodynamics of Intranasal Remimazolam-A Randomized Controlled Clinical Trial. Eur. J. Clin. Pharmacol. 76 (11), 1505-1516. doi:10.1007/s00228-020-02984-z

Pesic, M., Stöhr, T., Ossig, J., Borkett, K., Donsbach, M., Dao, V.-A., et al. (2020b). Remimazolam Has Low Oral Bioavailability and No Potential for Misuse in Drug-Facilitated Sexual Assaults, with or without Alcohol: Results from Two Randomised Clinical Trials. Drugs R. D 20 (3), 267-277. doi:10.1007/s40268020-00317-0

Probst, S., Bevilacqua, C., Eibel, S., Müller, A., Wahlers, S., Soehngen, M., et al. (2015). Difference in Vasopressor Use and Usage Patterns in Patients Undergoing Cardiac Surgery with Remimazolam versus Propofol/ Sevoflurane for General Anesthesia. Anesthesiology, A4025.

Probst, S., Eibel, S., Bevilacqua, C., Borkett, K., Soenghen, W., Soehngen, M., et al. (2014). Phase II Study of an Ultra-short Acting Benzodiazepine (Remimazolam) versus a Standard Regime of Propofol/Sevoflurane in Patients Undergoing Cardio-Surgery. Anesthesiology, A3038.

Rex, D. K., Bhandari, R., Desta, T., DeMicco, M. P., Schaeffer, C., Etzkorn, K., et al. (2018). A Phase III Study Evaluating the Efficacy and Safety of Remimazolam (CNS 7056) Compared with Placebo and Midazolam in Patients Undergoing Colonoscopy. Gastrointest. Endosc. 88 (3), 427-437.e6. doi:10.1016/ j.gie.2018.04.2351

Rex, D. K., Bhandari, R., Lorch, D. G., Meyers, M., Schippers, F., and Bernstein, D. (2021). Safety and Efficacy of Remimazolam in High Risk Colonoscopy: A Randomized Trial. Dig. Liver Dis. 53 (1), 94-101. doi:10.1016/j.dld.2020.10.039

Rudolph, U., Crestani, F., Benke, D., Brünig, I., Benson, J. A., Fritschy, J.-M., et al. (1999). Benzodiazepine Actions Mediated by Specific $\gamma$-aminobutyric acidA Receptor Subtypes. Nature 401 (6755), 796-800. doi:10.1038/44579

Rudolph, U., and Möhler, H. (2004). Analysis Ofgabaareceptorfunction Anddissection of Thepharmacology Ofbenzodiazepines
Andgeneralanestheticsthroughmousegenetics. Annu. Rev. Pharmacol. Toxicol. 44, 475-498. doi:10.1146/annurev.pharmtox.44.101802.121429

Saari, T. I., Uusi-Oukari, M., Ahonen, J., and Olkkola, K. T. (2011). Enhancement of GABAergic Activity: Neuropharmacological Effects of Benzodiazepines and Therapeutic Use in Anesthesiology. Pharmacol. Rev. 63, 243-267. doi:10.1124/ pr.110.002717

Sahinovic, M. M., Struys, M. M. R. F., and Absalom, A. R. (2018). Clinical Pharmacokinetics and Pharmacodynamics of Propofol. Clin. Pharmacokinet. 57 (12), 1539-1558. doi:10.1007/s40262-018-0672-3

Saito, K., Ohno, S., Maeda, M., Hirata, N., and Yamakage, M. (2021). Remimazolam Anesthesia for Cardiac Surgery with Cardiopulmonary Bypass: a Case Report. JA Clin. Rep. 7 (1), 21. doi:10.1186/s40981-021-00424-0

Sasaki, H., Hoshijima, H., and Mizuta, K. (2021). Ringer's Acetate SolutionInduced Precipitation of Remimazolam, Br. J. Anaesth. 126. e87-e89. doi:10.1016/j.bja.2020.11.021

Sato, T., Kato, Y., Yamamoto, M., and Nishiwaki, K. (2020). Novel Anesthetic Agent Remimazolam as an Alternative for the Asleep-Awake-Asleep Technique of Awake Craniotomy. JA Clin. Rep. 6 (1), 92. doi:10.1186/s40981-020-00398-5

Satoh, T., Nishihara, N., Sawashita, Y., Ohno, S., Hirata, N., and Yamakage, M. (2021). Remimazolam Anesthesia for MitraClip Implantation in a Patient with Advanced Heart Failure. Case Rep. Anesthesiology 2021, 1-4. doi:10.1155/2021/ 5536442

Savarese, J. J., Ali, H. H., Basta, S. J., Embree, P. B., Scott, R. P. F., Sunder, N., et al. (1988). The Clinical Neuromuscular Pharmacology of Mivacurium Chloride (BW B1090U). A Short-Acting Nondepolarizing Ester Neuromuscular Blocking Drug. Anesthesiology. United States 68 (5), 723-732. doi:10.1097/ 00000542-198805000-00010

Schippers, F., Pesic, M., Saunders, R., Borkett, K., Searle, S., Webster, L., et al. (2020). Randomized Crossover Trial to Compare Abuse Liability of Intravenous Remimazolam versus Intravenous Midazolam and Placebo in Recreational Central Nervous System Depressant Users. J. Clin. Pharmacol. 60 (9), 1189-1197. doi:10.1002/jcph.1614

Schüttler, J., Eisenried, A., Lerch, M., Fechner, J., Jeleazcov, C., and Ihmsen, H. (2020). Pharmacokinetics and Pharmacodynamics of Remimazolam (CNS 7056) after Continuous Infusion in Healthy Male Volunteers. Anesthesiology 132, 636-651. doi:10.1097/ALN.0000000000003103

Sheng, X.-Y., Liang, Y., Yang, X.-y., Li, L.-e., Ye, X., Zhao, X., et al. (2020). Safety, Pharmacokinetic and Pharmacodynamic Properties of Single Ascending Dose and Continuous Infusion of Remimazolam Besylate in Healthy Chinese Volunteers. Eur. J. Clin. Pharmacol. 76 (3), 383-391. doi:10.1007/s00228019-02800-3

Shioji, N., Everett, T., Suzuki, Y., and Aoyama, K. (2021). Pediatric Sedation Using Dexmedetomidine and Remimazolam for Magnetic Resonance Imaging. J. Anesth. doi:10.1007/s00540-021-02957-1

Sigel, E., and Ernst, M. (2018). The Benzodiazepine Binding Sites of GABAA Receptors. Trends Pharmacol. Sci. 39 (7), 659-671. doi:10.1016/ j.tips.2018.03.006

Simons, P. J., Cockshott, I. D., Douglas, E. J., Gordon, E. A., Hopkins, K., and Rowland, M. (1988). Disposition in Male Volunteers of a Subanaesthetic Intravenous Dose of an Oil in Water Emulsion of 14C-Propofol. Xenobiotica 18 (4), 429-440. doi:10.3109/00498258809041679

Sneyd, J. R., and Rigby-Jones, A. E. (2020). Remimazolam for Anaesthesia or Sedation. Curr. Opin. Anaesthesiol. 33, 506-511. doi:10.1097/ ACO.0000000000000877

Sokolowski, K. (2019). 'Remimazolam: PHARMACOLOGY/TOXICOLOGY NDA REVIEW and EVALUATION', FDA. Available at: https://www.accessdata.fda. gov/drugsatfda_docs/nda/2020/212295Orig1s000PharmR.pdf.

Soppitt, A. J., Glass, P. S. A., Howell, S., Weatherwax, K., and Gan, T. J. (2000). The Use of Propofol for its Antiemetic Effect: a Survey of Clinical Practice in the United States. J. Clin. Anesth. 12 (4), 265-269. doi:10.1016/s09528180(00)00151-3

Stafford, J. A., Pacofsky, G. J., Cox, R. F., Cowan, J. R., Dorsey, G. F., Gonzales, S. S., et al. (2002). Identification and Structure-Activity Studies of Novel UltrashortActing Benzodiazepine Receptor Agonists. Bioorg. Med. Chem. LettersEngland 12 (21), 3215-3218. doi:10.1016/s0960-894x(02)00512-7

Sternbach, L. H. (1979). The Benzodiazepine story. J. Med. Chem.United States 22 (1), 1-7. doi:10.1021/jm00187a001 
Suzuki, Y., Doi, M., and Nakajima, Y. (2021). General Anesthesia with Remimazolam in a Patient with Mitochondrial Encephalomyopathy: a Case Report. JA Clin. Rep. 7 (1), 51. doi:10.1186/s40981-021-00454-8

Tachibana, S., Hayamizu, K., and Yamakage, M. (2021). Remimazolam Use for Awake Craniotomy. JA Clin. Rep. 7, 25. doi:10.1186/s40981-021-00428-w

Tan, C. H., and Onsiong, M. K. (1998). Pain on Injection of Propofol. Anaesthesia 53 (5), 468-476. doi:10.1046/j.1365-2044.1998.00405.x

Tilbrook, G. S., and Bitt, L. J. (2006). 'Short Acting Benzodiazepine Salts and Their Polymorphic Forms', WO2008007071A1. Available at: https://patents.google. com/patent/WO2008007071A1/ko.

Tilbrook, G. S., and Kilpatrick, G. J. (2006). CNS 7056X, an Ultra-short Acting Benzodiazepine: In Vitro Metabolism. Anesthesiology 105, A543.

Tsurumi, K., Takahashi, S., Hiramoto, Y., Nagumo, K., Takazawa, T., and Kamiyama, Y. (2021). Remimazolam Anaphylaxis during Anesthesia Induction. J. Anesth. doi:10.1007/s00540-021-02934-8

Uhlén, M., Fagerberg, L., Hallstrom, B. M., Lindskog, C., Oksvold, P., Mardinoglu, A., et al. (2015). Tissue-based Map of the Human Proteome. Science 347 (6220), 1260419. doi:10.1126/science. 1260419

Upton, R. N., Martinez, A. M., and Grant, C. (2008). A Dose Escalation Study in Sheep of the Effects of the Benzodiazepine CNS 7056 on Sedation, the EEG and the Respiratory and Cardiovascular Systems. Br. J. Pharmacol. 155 (1), 52-61. doi:10.1038/bjp.2008.228

Upton, R. N., Martinez, A. M., and Grant, C. (2009). Comparison of the Sedative Properties of CNS 7056, Midazolam, and Propofol in Sheep. Br. J. Anaesth. 103 (6), 848-857. doi:10.1093/bja/aep269

Upton, R. N., Somogyi, A. A., Martinez, A. M., Colvill, J., and Grant, C. (2010). Pharmacokinetics and Pharmacodynamics of the Short-Acting Sedative CNS 7056 in Sheep. Br. J. Anaesth. 105 (6), 798-809. doi:10.1093/bja/aeq260

Wang, D., Zou, L., Jin, Q., Hou, J., Ge, G., and Yang, L. (2018). Human Carboxylesterases: a Comprehensive Review. Acta Pharmaceutica Sinica B 8 (5), 699-712. doi:10.1016/j.apsb.2018.05.005

Wang, W., and Sun, Q. (2020). Novel Targeted Drugs Approved by the NMPA and FDA in 2019. Sig Transduct Target. Ther. 5, 65. doi:10.1038/s41392-020-0164-4

Wang, X., Rida, N., Shi, J., Wu, A. H., Bleske, B. E., and Zhu, H.-J. (2017). A Comprehensive Functional Assessment of Carboxylesterase 1 Nonsynonymous Polymorphisms. Drug Metab. Dispos 45 (11), 1149-1155. doi:10.1124/ dmd.117.077669

Watanabe, T., Miyoshi, H., Noda, Y., Narasaki, S., Morio, A., Toyota, Y., et al. (2021). Effects of Remimazolam and Propofol on Ca2+ Regulation by Ryanodine Receptor 1 with Malignant Hyperthermia Mutation. Biomed. Res. Int. 2021, 1-11. doi:10.1155/2021/8845129

Wiltshire, H. R., Kilpatrick, G. J., Tilbrook, G. S., and Borkett, K. M. (2012). A Placebo- and Midazolam-Controlled Phase I Single Ascending-Dose Study Evaluating the Safety, Pharmacokinetics, and Pharmacodynamics of Remimazolam (CNS 7056). Anesth. Analgesia 115 (2), 284-296. doi:10.1213/ ANE.0b013e318241f68a

Wood, M., and Stark, R. (2018). John (Iain) Glen Wins 2018 Lasker Prize for Development of Propofol. Anesthesiology 129, 1055-1056. doi:10.1097/ ALN.0000000000002481

Worthington, M. T., Antonik, L. J., Goldwater, D. R., Lees, J. P., Wilhelm-Ogunbiyi, K., Borkett, K. M., et al. (2013). A Phase Ib, Dose-Finding Study of Multiple Doses of Remimazolam (Cns 7056) in Volunteers Undergoing Colonoscopy. Anesth. Analgesia 117 (5), 1093-1100. doi:10.1213/ANE.0b013e3182a705ae

Xie, H., Lu, F., Liu, W., Wang, E., Wang, L., and Zhong, M. (2021). Remimazolam Alleviates Neuropathic Pain via Regulating Bradykinin Receptor B1 and Autophagy. J. Pharm. Pharmacol. rgab080. doi:10.1093/jpp/rgab080
Xu, W., Hu, J., Liu, W., Zhu, Q., Gong, X., Zhu, P., et al. (2021). Remimazolan Inhibits Glioma Cell Growth and Induces Apoptosis through Down-regulation of NF-кB Pathway. IUBMB Life 73 (2), 341-348. doi:10.1002/iub.2433

Yamamoto, T., Kurabe, M., and Kamiya, Y. (2021a). A Mechanism of Re-sedation Caused by Remimazolam. J. Anesth. 35, 467-468. doi:10.1007/s00540-021-02930-y

Yamamoto, T., Kurabe, M., and Kamiya, Y. (2021b). Re-sleeping after Reversal of Remimazolam by Flumazenil. J. Anesth. 35, 322. doi:10.1007/s00540-021-02915-x

Yoshida, A., Kurata, S., Kida, K., and Tsubokawa, T. (2021a). Anesthetic Management for the Sleep-Awake-Sleep Technique of Awake Craniotomy Using a Novel Benzodiazepine Remimazolam and its Antagonist Flumazenil. JA Clin. Rep. 7 (1), 14. doi:10.1186/s40981-021-00417-z

Yoshida, K., Tanaka, S., and Watanabe, K. (2021b). A Case of Intravenous Line Occlusion when Using Acetated Ringer's Solution and Remimazolam. J. Clin. Anesth. 70, 110190. doi:10.1016/j.jclinane.2021.110190

Yoshikawa, H., Hosokawa, M., Kashima, Y., Oki, S., and Masui, K. (2021) Remimazolam Tolerance in Long-Term Benzodiazepine Users: A Case Report of 2 Cases. A A Pract., 15(5, e01460), p. doi: doi:10.1213/ XAA.0000000000001460

Yuan, R., Flockhart, D. A., and Balian, J. D. (1999). Pharmacokinetic and Pharmacodynamic Consequences of Metabolism-Based Drug Interactions with Alprazolam, Midazolam, and Triazolam. J. Clin. Pharmacol. Engl. 39 (11), 1109-1125. doi:10.1177/00912709922012006

Zhang, X., Li, S., and Liu, J. (2021) Efficacy and Safety of Remimazolam Besylate versus Propofol during Hysteroscopy: Single-centre Randomized Controlled Trial. Available at:doi: doi:10.21203/rs.3.rs-209379/v1

Zhou, J., Curd, L., Lohmer, L. L., Ossig, J., Schippers, F., Stoehr, T., et al. (2020b). Population Pharmacokinetics of Remimazolam in Procedural Sedation with Nonhomogeneously Mixed Arterial and Venous Concentrations. Clin. Transl Sci. 14, 326-334. doi:10.1111/cts. 12875

Zhou, J., Curd, L., Lohmer, L. R. L., Delpratt, N., Ossig, J., Schippers, F., et al. (2021). A Population Pharmacodynamic Markov Mixed-effects Model for Determining Remimazolam-induced Sedation when Co-administered with Fentanyl in Procedural Sedation. Clin. Transl Sci. doi:10.1111/cts.13023

Zhou, J., Leonowens, C., Ivaturi, V. D., Lohmer, L. L., Curd, L., Ossig, J., et al. (2020a). Population Pharmacokinetic/pharmacodynamic Modeling for Remimazolam in the Induction and Maintenance of General Anesthesia in Healthy Subjects and in Surgical Subjects. J. Clin. Anesth. 66, 109899. doi:10.1016/j.jclinane.2020.109899

Zhou, Y., Hu, P., Huang, Y., Sang, N., Song, K., Wang, H., et al. (2018). Population Pharmacokinetic/Pharmacodynamic Model-Guided Dosing Optimization of a Novel Sedative HR7056 in Chinese Healthy Subjects. Front. Pharmacol. 9, 1316. doi:10.3389/fphar.2018.01316

Zhou, Y., Hu, P., and Jiang, J. (2017). Metabolite Characterization of a Novel Sedative Drug, Remimazolam in Human Plasma and Urine Using Ultra HighPerformance Liquid Chromatography Coupled with Synapt High-Definition Mass Spectrometry. J. Pharm. Biomed. Anal. 137, 78-83. doi:10.1016/ j.jpba.2017.01.016

Conflict of Interest: The author is a director and employee of GJK Pharma Ltd.

Copyright (c) 2021 Kilpatrick. This is an open-access article distributed under the terms of the Creative Commons Attribution License (CC BY). The use, distribution or reproduction in other forums is permitted, provided the original author $(s)$ and the copyright owner(s) are credited and that the original publication in this journal is cited, in accordance with accepted academic practice. No use, distribution or reproduction is permitted which does not comply with these terms. 